\title{
A Review of Underwater Localization Techniques, Algorithms, and Challenges
}

\author{
Xin Su $\mathbb{D},{ }^{1}$ Inam Ullah $\mathbb{D},{ }^{1}$ Xiaofeng Liu, ${ }^{1}$ and Dongmin Choi $\mathbb{D}^{2}$ \\ ${ }^{1}$ The College of Internet of Things (IoT) Engineering, Hohai University (HHU), Changzhou 213022, China \\ ${ }^{2}$ The Division of Undeclared Majors, Chosun University, Gwangju 61452, Republic of Korea \\ Correspondence should be addressed to Dongmin Choi; jdmcc@chosun.ac.kr
}

Received 14 October 2019; Revised 26 November 2019; Accepted 3 December 2019; Published 13 January 2020

Guest Editor: Yuan Li

Copyright ( 92020 Xin Su et al. This is an open access article distributed under the Creative Commons Attribution License, which permits unrestricted use, distribution, and reproduction in any medium, provided the original work is properly cited.

\begin{abstract}
Recently, there has been increasing interest in the field of underwater wireless sensor networks (UWSNs), which is a basic source for the exploration of the ocean environment. A range of military and civilian applications is anticipated to assist UWSN. The UWSN is being developed by the extensive wireless sensor network (WSN) applications and wireless technologies. Therefore, in this paper, a review has been presented which unveils the existing challenges in the underwater environment. In this review, firstly, an introduction to UWSN is presented. After that, underwater localizations and the basics are presented. Secondly, the paper focuses on the architecture of UWSN and technologies used for underwater acoustic sensor network (UASN) localization. Various localization techniques are discussed in the paper classified by centralized and distributed localizations. They are further classified into estimated and prediction-based localizations. Also, various underwater localization algorithms are discussed, which are grouped by the algorithms based on range and range-free schemes. Finally, the paper focuses on the challenges existing in underwater localizations, underwater acoustic communications with conclusions.
\end{abstract}

\section{Introduction}

Underwater wireless sensor networks (UWSNs) have shown increasing interest, in the latest years. For a variety of applications, underwater sensor networks (USNs) can be implemented. Each implementation is essential in its domain, but some of them can enhance ocean exploration to meet the variety of underwater applications, including a natural disaster alert scheme (i.e., tsunami and seismic tracking), aided navigation, oceanographic information collection, and underwater surveillance, ecological applications (i.e., quality of biological water, tracking of pollution), industrial applications (i.e., marine exploration), etc. For example, for offshore engineering applications, sensors can assess certain parameters such as base intensity and mooring tension to monitor the structural quality of the mooring environment [1]. Underwater acoustic sensors networks (UASNs) provide a new platform for under communication to explore the underwater environment. UASNs have also improved the understanding related to underwater environments such as climate changes, animal life in underwater, and the population of coral reefs.

In [2], the authors present a localization technique for UASN in which the mobility of the sensor node is considered and all the unknown sensor nodes are successfully placed at different positions. The positioning system is recursive and the localization method involves distinct sensor nodes. UASNs also increase the underwater warfare capabilities of the naval forces so that they can be used for the detection of a submarine, unmanned operation system, surveillance, and mine countermeasure algorithms. UASNs can also help monitor or control the oil rigs that can take prevent the disaster's effects such as rigs explosion in the Gulf of Mexico once occurred (2010). Similarly, UASN technology also helps in tsunami and earthquake forewarning. A unique system is called 3-DUL, which originally consisted of only three anchor sensor nodes, such as buoys on the water surface, which defuse their worldwide position data in all three directions and 3-DUL follows a 2-phase operation [3-6]. The distances to nearby anchor nodes are determined by a node with 
TABLE 1: Comparison table of electromagnetic, acoustic, and optical waves in underwater environment.

\begin{tabular}{lccr}
\hline & Electromagnetic waves & Acoustic waves & Optical waves \\
\hline Frequency band & $\sim \mathrm{kHz}$ & $\sim \mathrm{MHz}$ & $\sim 10^{14}-10^{15} \mathrm{~Hz}$ \\
Bandwidth & $\sim \mathrm{kHz}$ & $\sim \mathrm{MHz}$ & $\sim 10-150 \mathrm{MHz}$ \\
Power loss & $>0.1 \mathrm{~dB} / \mathrm{m} / \mathrm{Hz}$ & $\sim 28 \mathrm{~dB} / 1 \mathrm{~km} / 100 \mathrm{MHz}$ & $\propto$ turbidity \\
Effective range & $\sim 1 \mathrm{~m}$ & $\sim 10 \mathrm{~m}$ & $\sim 10-100 \mathrm{~m}$ \\
Nominal speed $(\mathrm{m} / \mathrm{s})$ & $\sim 1,500$ & $\sim 33,333,333$ & $\sim 33,333,333$ \\
Antenna size & $\sim 0.1 \mathrm{~m}$ & $\sim 0.5 \mathrm{~m}$ & $\sim 0.1 \mathrm{~m}$ \\
\hline
\end{tabular}

an unknown place during the first stage. The anchor nodes are projected to their horizontal level in the second phase and form a virtual geometric shape using the depth information from these multivariate ranges. If the corresponding shape is robust, the sensor node will find itself and become an anchor sensor node through the dynamic trilateration method. In three-dimensional (3D) topology, this method iterates dynamically in all directions to locate as many sensors as possible. A 3D localization method takes into account the attenuation of electromagnetic (EM) waves over the reliable elevation angle spectrum. They pick the radiation patterns of dipole antennas to determine the reliable elevation range. The feasibleness of this scheme is presented in distance estimation and 3D localization schemes by changing the elevation angle and distance. However, in [7], the writers suggest a fresh model that utilizes the benefits of the features of EM waves in water. The sensor node cannot only evaluate the distance with low environmental noise but also ensure precise localization output with elevated sampling rates. Using the sets of RF sensors, a UWSN is built for this localization system at the target docking location. A 3D underwater localization algorithm is also suggested in [8] for a marine near-sea surveillance scheme that utilizes a tiny amount of beacons for localization. Performance evaluations show that the worldwide localization of three surface anchor nodes is effectively spread by 3DUL. Its simple algorithm makes it possible for UASNs to adapt to the vibrant nature of the water globe $[9,10]$. To this end, the ocean surveillance system is used that can gather information from the ocean and its surrounding areas and provide this information via satellite communication to the ship or on-shore center or sometimes use underwater wires. These are replaced by less expensive and small underwater nodes that use this equipment in UASN to house various nodes on board, such as pressure, temperature, and salinity. Underwater sensor nodes are networked and can interact using acoustic signals.

As we know, in underwater the radio signals can only travel to a short distance because the radio signal attenuates highly underwater and optical signals cannot travel in an inappropriate medium because of the dispersion of the optical signals. An acoustic signal scatters less as compared to radio and optical signals, resulting in an acoustic signal being more useful for underwater communication purposes as compared to radio and optical signals as shown in Table 1. However, the acoustic bandwidth in the underwater is smaller, resulting in reduced information rates. Multiple sensor nodes are needed to raise information rates and have short-range communication, resulting in excellent coverage. The acoustic channel also has a low quality of connection $[11,12]$ owing to the time variability of the propagation of the medium and multipath. The underwater sound speed is approximately $1500 \mathrm{~m} / \mathrm{s}$, resulting in very elevated delay propagation. The UASNs are also energy-limited as WSN due to these difficulties. Also, localization is a fundamental task that is used to detect the location of a target in the underwater medium for various purposes such as data tagging, tracking nodes in the underwater and coordinating the movement of node groups. For the tracking of a target [6, $13,14]$, research work proposes a semidefinite programming- (SDP-) based localization procedure that is achieved by measurements obtained via onboard pressure sensors. SDP enhances the point localization precision and provides quicker convergence for monitoring under the same system setup and environmental circumstances, particularly at low signal to noise ratio (SNR). On condition that geomagnetic anomaly can be reversed as a magnetic dipole target, the localization of an underwater vessel relative to the target is calculated by the magnitude of the magnetic field and target gradients. The magnetic field is calculated by the device mounted on a car comprised of ten magnetometers of one axis. Since the noise of magnetometers results in the coefficients of a six-order formula [15] with an unsuitable element, the localization accuracy will be influenced by the weather. In [16], USNs can modify ocean exploration to enable a list of new applications that are not presently feasible or expensive to implement, including oceanographic information collection, ecological applications, government security, underwater military tracking, and commercial operation.

For maritime defense purposes, USNs can provide immediate deployment and enhance coverage in coastal area surveillance applications. USNs mounted on the bottom of the ocean with underwater sensor nodes can detect earthquakes and tsunami formations before entering residential areas. A rough drawing of underwater node operation is shown in Figure 1. Mobile USNs can track polluted waters for water pollution detection devices as they propagate to clean water from their source and warn authorities to take action. USNs could be used to monitor coastal creatures and coral reefs, where there is limited data about human activity. The Gaussian noise injection detector (GNID) is proposed [17], to improve the probability of detection based on the noiseenhanced signal detection using a prewhitening filter, time frequency denoising technique with $S$-transform, in inverse whitening filter results in improving underwater signal 

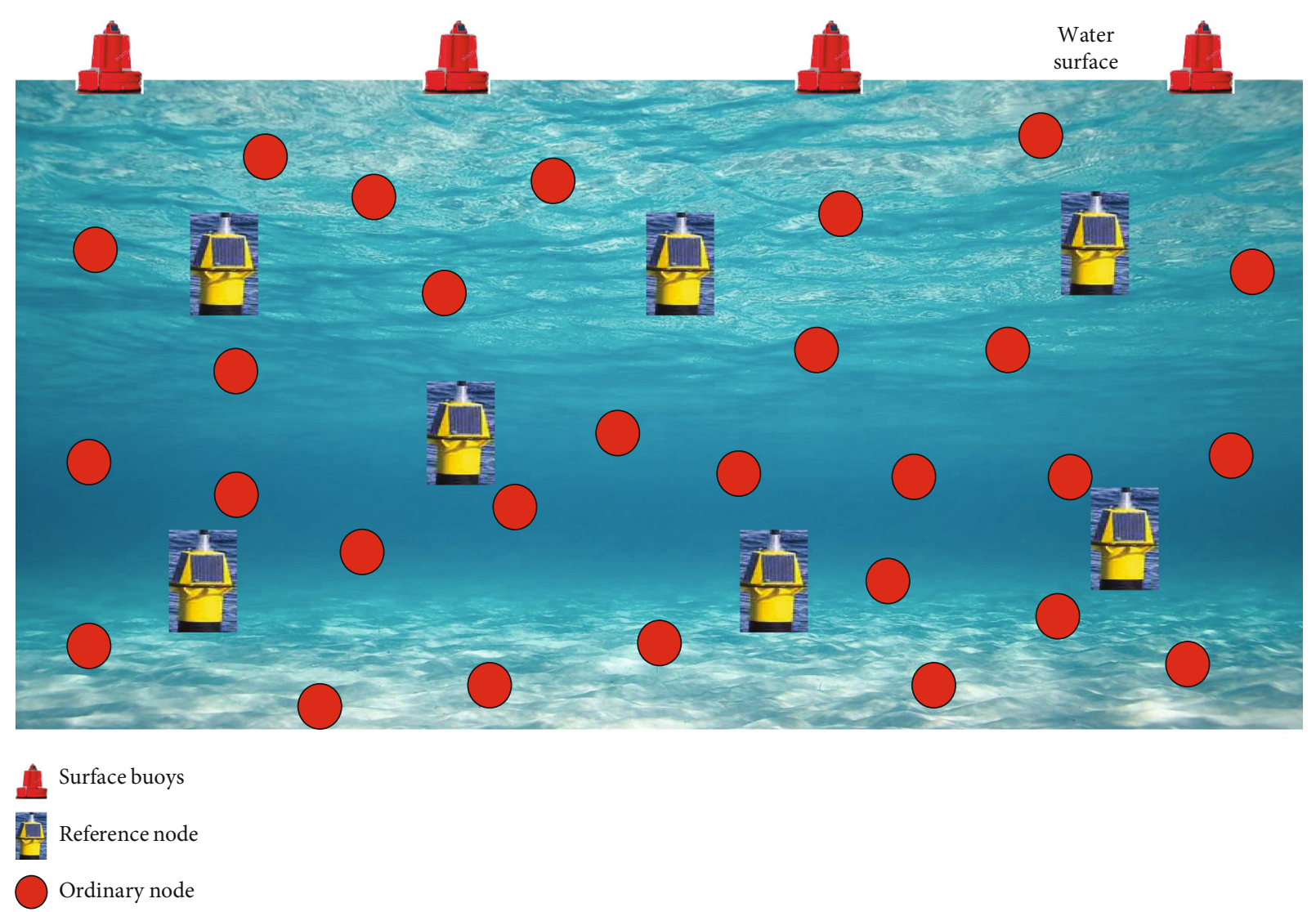

Figure 1: A rough sketch of the underwater node deployment.

detection. Environmental monitoring is also a vibrant aspect of determining safety and health problems for environmental or mankind's health. Environmental monitoring's main purpose is sampling soil, water, and atmospheric but they also need to take the air samples inside buildings to guarantee rules are met. The group of people working in environmental monitoring needs to looking for many things which are important in fact. The most obvious is the radioactivity or pollutants, especially in the case when looking to build a case of negligence against a distinct or for evidence of the effects of changes in climate.

The key objectives of this paper are to outlines a comprehensive review of underwater localization techniques and their algorithms. We attempt to review the different aspects of underwater communications and underwater localizations by considering various attributes. The main objective of this review is to provide a detailed knowledge of underwater localization techniques, localization algorithms, architecture, etc. We also highlight the weaknesses and strengths of the existing underwater localization techniques that can help the researchers to identify more efficient and accurate solutions for the existing challenges. Explicitly, this review aims to answer the following basic questions:

(i) What are the state-of-the-art localization techniques?

(ii) What are the main research challenges in underwater localization? (iii) What are the main defenses and their pros and cons?

(iv) What are the promising solutions to improve underwater localization?

This review makes the following contributions:

(i) This review provides a detail explanation of localization for the underwater environment which covers the localization basics, architecture, localization techniques, and algorithms used for localization

(ii) This review rises the basic requirements for localization such as security attacks on underwater nodes

(iii) Based on a detailed analysis of existing underwater localization techniques, we presented the existing challenges and future directions that need to be considered in the recent future

The paper is organized as follows. Section 2 presents the procedure and basics of localization. Next, Section 3 presents the architecture of UASN, Section 4 presents the related works, and Section 5 presents the techniques used for UASN localizations. Furthermore, Section 6 and Section 7 present range-based and range-free algorithms for localization, respectively. Section 8 presents the performance evaluation of underwater localization schemes. Finally, Section 9 presents the existing challenges and open issues in UWSNs and Section 10 concludes the paper. 


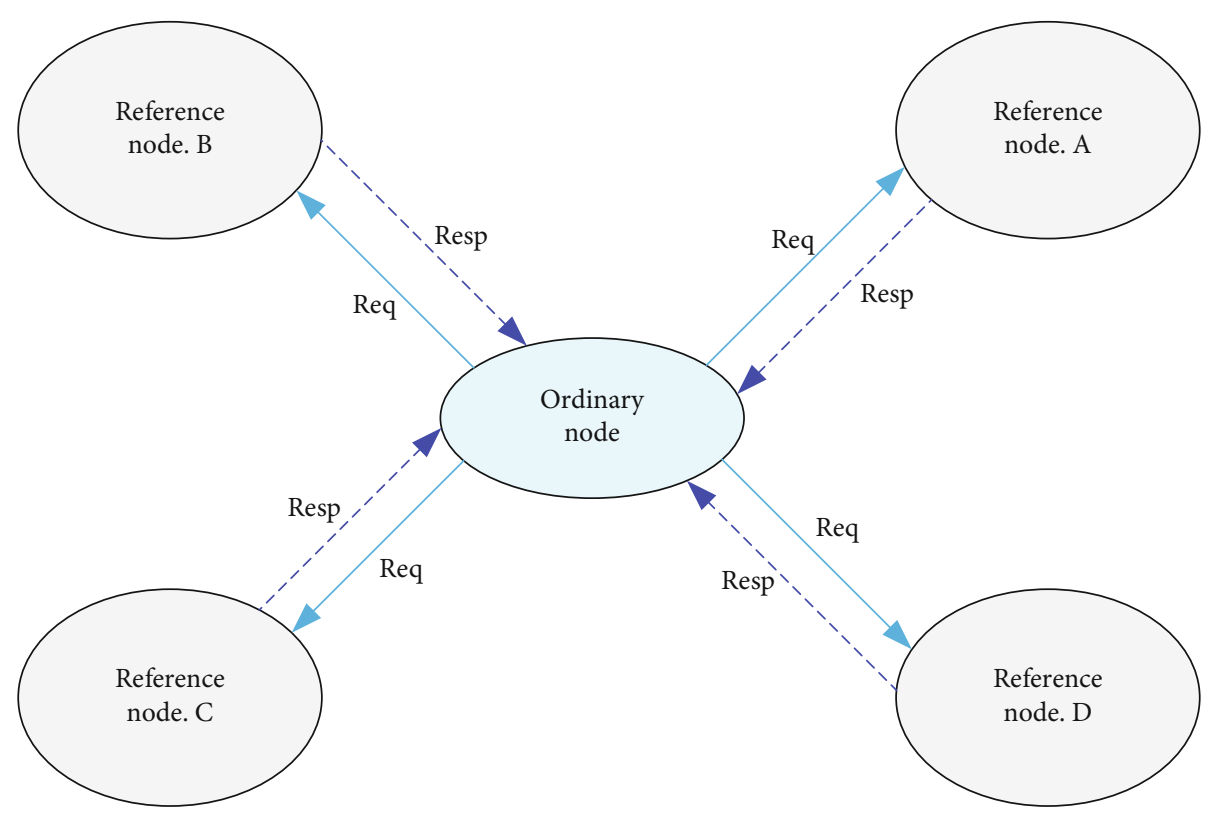

FIgure 2: Two-way message exchange of reference and ordinary node.

\section{Procedure and Basics of Localization}

Assumptions for the localization operation need to take care of is that all antenna nodes have an ideal understanding of their position and should share clock information with the other sensor nodes worldwide synchronization. All nodes should meet to share data at any time, i.e., every individual sensor node can retrieve all readings and execute the process of localization before carrying all data back to the active or reference nodes. Each sensor node in the corresponding destination frame can communicate completely with other nodes and has no accident or interference problems [18]. At time $t_{0}$, the active sensor node emits a single message requesting location through all of the listening nodes and each node gets message at the time $t_{n, n}$. On the given message, each node conducts a Doppler speed estimate. After gathering all data from sensors, a master node gets all estimates and performs the operation of localization and transmits the complete estimate back to the active node. Alternatively, it is possible to collect and relay the data to the active node where localization is done after that. The updates of the active node or master node estimate tracking and navigation algorithms as estimates of points are acquired. Localization is another difficult work; the use of Global Positioning System (GPS) is limited to surface nodes because in underwater, GPS signal does not propagate [19]. Alternative GPS fewer positioning approaches for terrestrial sensor networks have been provided, but they must be amended owing to the description of the acoustic channel. The acoustic channel has low bandwidth, high delay in propagation, and high error rate. Localization protocols, therefore, need to operate with the minimal feasible exchange of messages between nodes and exchange of messages such as two-way data exchange (see in Figure 2). This is also assessed by the sensor node's restricted battery energy and the underwater sensor node battery recharge or replacement problem.

To execute localization in a better way, it requires several objects with known locations, i.e., anchors and distance or angle measurement between anchors and the object to be located underwater, i.e., unknown sensor node. The anchors can be placed in a fixed position and their coordinates may have been configured in the beginning, or they may have distinctive hardware to learn from the location server such as GPS. Using angle or distance measurement between the anchor and the unknown node to estimate the location of an unknown sensor node and also combining measurements occur. Sensors presently used for oceanographic studies are either located with long or short baseline (LBL/SBL) devices. With a set of receivers based on acoustic wave communication, sensor positions are described in both instances. Acoustic transponders are introduced in the LBL either on the seabed or underfloor moorings around the application region $[20,21]$. The effects of multiple error sources on the LBLbased scheme's localization accuracy have been investigated and evaluated in detail. It shows that the more severe variables that may affect general accuracy are the calibration of the transmitters and the amounts of data about the sound speed in the operating area. A vessel follows the sensors in the SBL scheme and utilizes a short-range emitter to allow the process of localization.

UWSN is characteristically composed of various nodes that are anchored to the lowermost of the ocean wirelessly linked with underwater gateways [22-24]. The information from these sensors is transmitted within this network from the lowermost of the sea to the water surface station by applying multihop links. The gateways in underwater are furnished with definite nodes with both upright and straight transceivers. The first gateway is utilized to transfer instructions and constellation information to the nodes and receive 


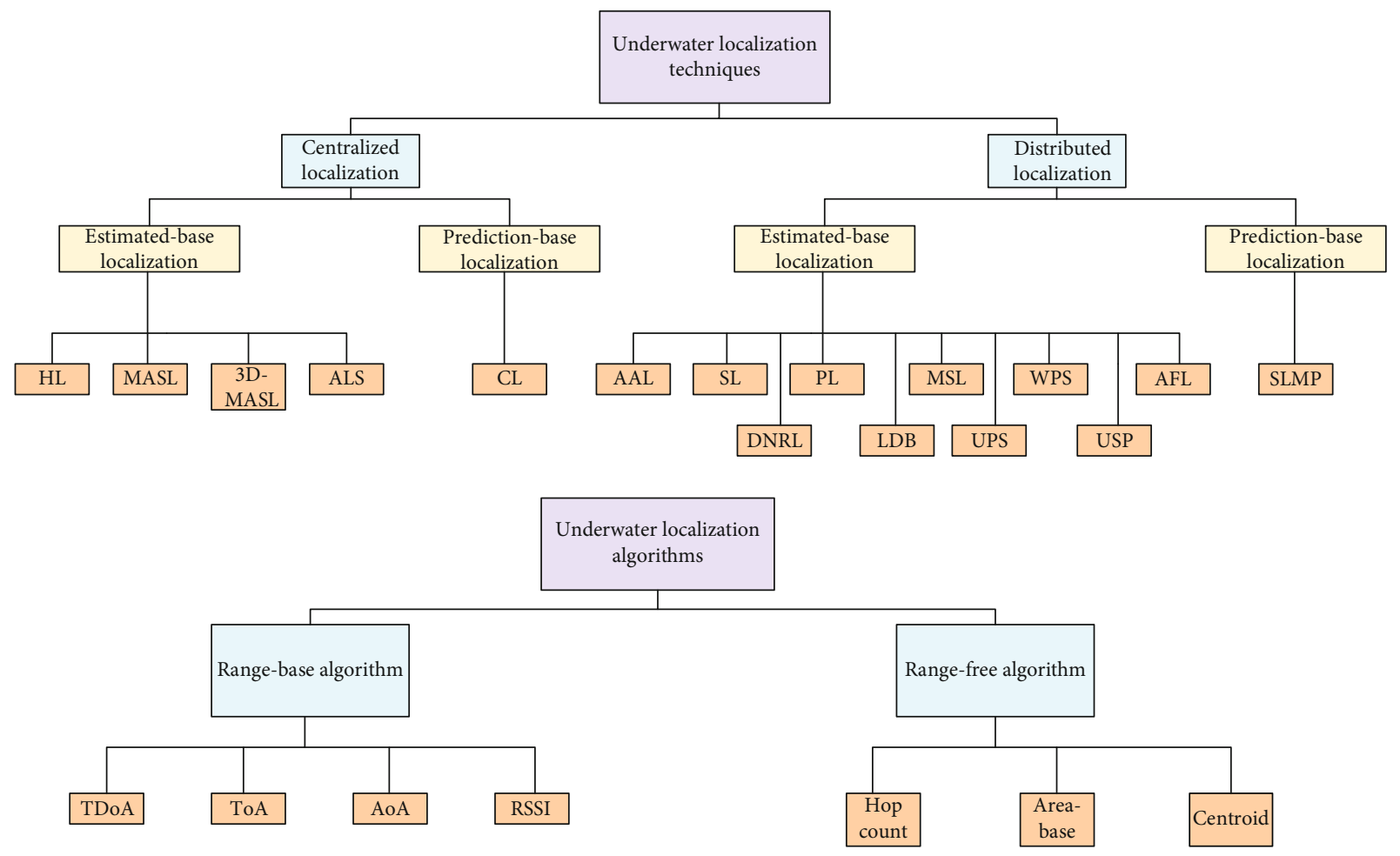

Figure 3: Architecture of underwater localization techniques and underwater localization algorithms.

the collected information back from the node. The second gateway is used to transmit the supervised information to the water surface station. Contrasting shallow water, upright communication is typically essential for a long range in deep water to attain the transfer of information to the water surface station. The acoustic communication is applied to accomplish multiple communications to collect the information from the nodes, where radio communication is generally conventional with satellite communication to transmit the collected information to the coastal sink. The underwater sensor nodes in the propagation range will sense a series of transmissions and decode packets. To compare the receiving time with the transmission time encoded in the packet, every sensor node can achieve the time of arrival (ToA) estimates of the packet message from various surface nodes, based on which it tries to calculate its particular location. The broadcast from the surface nodes to underwater nodes is one-way communication and the quality of localization is independent of the number of sensor nodes in underwater, and there is no extra interference between underwater sensor nodes. The underwater sensor nodes contain a controller to accommodate with an oceanographic sensor through a sensor pledge micro security [25]. The receiving data from the sensor through the controller is stored in the onboard memory. The controller can store, progress, and broadcast to the network devices.

\section{Architecture of UASN}

It is well known that energy consumption is more important in UWSN, which may limit long life cycles. Therefore, the network topology is the basic aspect that needs to be carefully designed to reduce the serious impact on network performance. Also, the reliability and capacity of the network depend on the network topology. Therefore, how to organize such a network topology is a challenging task, and researchers need to pay more attention to network topology. Here, the architecture of UASN is classified according to two metrics: one is the motion capability of the sensor nodes, i.e., stationary, mobile, or hybrid; the other is the spatial coverage of UASN, i.e., 2D or 3D UASNs as shown in Figure 3. The nodes float freely under the water in the portable UASNs with unpropelled and untethered sensor nodes and drift with the water current. In UASN with powered sensor nodes, the node motion can be controlled by inertial navigation systems. Autonomous Underwater Vehicles (AUVs) and Unmanned Underwater Vehicles (UUV) are floats, drifters, gliders, and profiling float, along with examples of unpropelled portable machinery. Most of these instruments are used in oceanography to gather data and measurements from the various layers of the ocean environment. Drifters operate mostly on the ground and drift with winds and surface waves as floats move with the current of the water. They are used to acquire measurements from the surface of the ocean and send the information to the on-shore center via satellite or GPS. Gliders are devices driven by buoyancy, as they can travel vertically comparable to floats for profiling. Besides that, with the assistance of their body and wing design structure, they can move horizontally. Sensor nodes are linked to surface buoys or ocean floor units having a fixed position in the stationary UASNs. For example, the port entrance, stationary UASNs are applied for controlling a certain region. Mobile and stationary nodes coexist in hybrid UASN architectures. In $[26,27]$, using a hybrid architecture, 
a portable sink node transverses the network and collects data from underwater sensor nodes.

The immediate domain with acoustic energy density is comparable to the density of the acoustic energy source in the resounding field and an open atmosphere with an acoustic energy density using acoustic waves such as $W_{r}$ reflecting the various wall. Direct field acoustic energy density depends on the distance $r$ to the source associated with:

$$
W_{d}(r)=\left(\frac{P_{s}}{4 \pi c r^{2}}\right),
$$

where $P_{s}$ is the source force and $c$ is the sound velocity. It implicitly assumes an omnidirectional source; if the source is directional, the direct field relies on the direction.

And the acoustic energy density $W_{r}$ and the acoustic intensity $I_{r}$ are linked to an isotropic homogenous reverberated domain [28] by:

$$
W_{r}=\left(\frac{4 I_{r}}{c}\right) \text {. }
$$

The derivative of the complete acoustic power in the tank, $Q=W_{r}(V)$, is the variance between the acoustic power that is forced by the $P_{s}$ source and the acoustic power is degenerated by the absorption or transmission on the wall $a I_{r}$.

$$
\frac{d Q}{d t}=P_{s}-a I_{r}=P_{s}-\frac{a c}{4 V} Q
$$

In Equation (3), $a$ is the Sabine coefficient which represents the absorption and transmission due to the walls. When the balance is reached $(d Q / d t)=0$, then

$$
P_{s}=\frac{a c}{4 V} Q=\frac{a c}{4} W_{r}
$$

It represents the belonging between the source power $P_{s}$ and the density of acoustic energy in the reverberation sector $W_{r}$.

In 2D UASNs, all sensor nodes are supposed to be on the same depth, e.g., they can be deployed on the seafloor and ocean surface, and each sensor node can float at an arbitrary depth in 3D UASNs [29]. Stationary sensor networks are generally regarded to be $2 \mathrm{D}$ as the sensor nodes are positioned on the ground buoys or anchors of the ocean floor. The wealth of UASN architecture is due in part to a conventional description of UASNs and in part to its application and particular design criteria. For example, GPS can be used for a 2D stationary UASN with nodes deployed on the sea surface, or for similar UASNs with ocean floor units, the sensor nodes can be deployed in predefined location is trivial. Furthermore, stationary UASNs do not involve regular localization as do portable UASNs, which implies that localization protocols with comparatively elevated overhead communication can still be used as they only operate at the moment of configuration. In [30], the authors also proposed the architecture of UASNs and divided it into different groups such as (a) static architecture, (b) hybrid architectures, and (c) mobile UASNs and free-floating networks.

\section{Related Works}

In this section, we give a brief description of underwater acoustic communication. After that, we review some widely known schemes which are used for underwater localization. Currently, the underwater communication system utilizes EM, optics, and acoustic data transmission schemes to transfer data among the various locations of the nodes. EM communication scheme is influenced by the conducting nature of the underwater environment while optic waves are only able to move on very short distance because optic waves are easier to absorb in underwater environments $[31,32]$. Therefore, an acoustic communication scheme is only one scheme that has better performance as compared to EM and optical due to less attenuation in the underwater environment. Acoustic signals also have less attenuation in the deep and thermally stable underwater field. Acoustic signals attenuate more in shallow as compared to deep water because of the temperature, noise, and multipath reflection and refraction. In the underwater field, the sound speed is not constant instead of this sound speed varies almost at every point. Near to the water surface, sound speed is almost 1500 that is four times higher than the sound speed in air and very slow as compared to the EM and optic speed in air.

Due to the unique challenges of the acoustic channel, it is highly variant, for example, high propagation delay, variable sound speed, narrow bandwidth, reflection, and refraction. Because of these unique properties, it creates more issues regarding MAC protocols. MAC protocols have two main groups such as content-based and scheduledbased protocols. Content-based nodes complete each other for the exchange of signals, while scheduled-based avoid collision among the transmission nodes. Content-based are not suitable for the underwater environment, while scheduledbased such as TDMA and FDMA are not efficient due to the high propagation delay and narrow bandwidth, respectively; however, CDMA is appropriate for UANs $[33,34]$.

A localization scheme for UWSN is presented for localization issues in large-scale UWSNs. Unlike in TWSN, GPS cannot work properly in underwater or attenuate highly [35]. Due to the costly equipment of underwater, limited bandwidth and harshly impaired channel all make the process of localization very challenging. Currently, most of the localization techniques are not well appropriate for the deep underwater field. The researchers presented a new scheme that mainly consists of four types of sensor nodes, such as DETs, surface buoys, ordinary nodes, and anchor nodes. DET is connected to the surface buoys and can dive and rise to the water surface for the broadcasting of its location. Surface buoys are supposed to connect with the GPS. Anchor node can estimate their locations based on location information from the DETs and estimation of the distance to the DETs. This localization scheme is scalable and can be applied to make balances on the accuracy and cost of localization.

In [36], the authors have presented a new SLMP localization technique with the prediction of mobility, for the large- 
TABLE 2: Performance evaluation of underwater acoustic networks and underwater localization schemes.

\begin{tabular}{|c|c|c|c|c|c|c|c|c|c|c|}
\hline Ref. & $\begin{array}{c}\text { Energy } \\
\text { consumption }\end{array}$ & $\begin{array}{l}\text { Network } \\
\text { lifetime }\end{array}$ & $\begin{array}{l}\text { No. of } \\
\text { nodes }\end{array}$ & $\begin{array}{c}\text { Time } \\
\text { synchronization }\end{array}$ & $\begin{array}{c}\text { Packet } \\
\text { exchange rate }\end{array}$ & $\begin{array}{c}\text { Loca. } \\
\text { accuracy }\end{array}$ & $\begin{array}{c}\text { Comm. } \\
\text { overhead }\end{array}$ & Delay & $\begin{array}{c}\text { Error } \\
\text { estimation }\end{array}$ & $\begin{array}{c}\text { Link } \\
\text { quality }\end{array}$ \\
\hline [3] & $\mathrm{X}$ & $\checkmark$ & $\checkmark$ & - & $\checkmark$ & $\checkmark$ & $X$ & $\checkmark$ & $\checkmark$ & $\checkmark$ \\
\hline [18] & - & - & $\checkmark$ & $\mathrm{X}$ & $\checkmark$ & $\checkmark$ & - & - & $\checkmark$ & - \\
\hline [21] & $\checkmark$ & X & $\mathrm{X}$ & $\mathrm{X}$ & $\mathrm{X}$ & $\checkmark$ & - & $X$ & $\checkmark$ & $\mathrm{X}$ \\
\hline [26] & $\mathrm{X}$ & - & $\checkmark$ & $\checkmark$ & $\checkmark$ & $\mathrm{X}$ & $\checkmark$ & $\checkmark$ & $\mathrm{X}$ & - \\
\hline [41] & $\mathrm{X}$ & - & $\checkmark$ & - & $\checkmark$ & $\checkmark$ & - & $\checkmark$ & $\mathrm{X}$ & - \\
\hline [42] & - & $\mathrm{X}$ & $\mathrm{X}$ & $\checkmark$ & $\mathrm{X}$ & $\checkmark$ & $\mathrm{X}$ & - & $\checkmark$ & - \\
\hline [43] & $\mathrm{X}$ & - & $\checkmark$ & $\checkmark$ & $\checkmark$ & $\mathrm{X}$ & - & $\mathrm{X}$ & $\checkmark$ & - \\
\hline [44] & - & - & $\mathrm{X}$ & $\checkmark$ & $\checkmark$ & $\checkmark$ & $\checkmark$ & - & $\checkmark$ & $\mathrm{X}$ \\
\hline [46] & $\mathrm{X}$ & $\checkmark$ & $\checkmark$ & - & $\checkmark$ & $\checkmark$ & - & $\checkmark$ & $\checkmark$ & - \\
\hline [47] & $\mathrm{X}$ & $\checkmark$ & $\checkmark$ & - & $\checkmark$ & $\checkmark$ & $\mathrm{X}$ & $\mathrm{X}$ & $\mathrm{X}$ & $\checkmark$ \\
\hline [37] & $\mathrm{X}$ & $\checkmark$ & $\checkmark$ & $\checkmark$ & $\checkmark$ & $\checkmark$ & $\mathrm{X}$ & $\mathrm{X}$ & $\mathrm{X}$ & $\checkmark$ \\
\hline [48] & $\mathrm{X}$ & - & $\checkmark$ & - & $\checkmark$ & $\checkmark$ & $\mathrm{X}$ & X & $\mathrm{X}$ & $\checkmark$ \\
\hline [49] & $\mathrm{X}$ & - & $\checkmark$ & $\checkmark$ & $\checkmark$ & - & - & $\checkmark$ & - & $\mathrm{X}$ \\
\hline [51] & $\mathrm{X}$ & $\mathrm{X}$ & $\checkmark$ & $\mathrm{X}$ & - & $\mathrm{X}$ & $\mathrm{X}$ & - & $\checkmark$ & - \\
\hline [53] & $\mathrm{X}$ & $\checkmark$ & $\checkmark$ & $\mathrm{X}$ & $\checkmark$ & $\checkmark$ & $\mathrm{X}$ & $\mathrm{X}$ & $\checkmark$ & $\checkmark$ \\
\hline [56] & $\mathrm{X}$ & - & $\checkmark$ & - & $\checkmark$ & $\checkmark$ & - & $\checkmark$ & $\checkmark$ & - \\
\hline [57] & $\checkmark$ & - & $\checkmark$ & $\mathrm{X}$ & $\checkmark$ & $\mathrm{X}$ & $\mathrm{X}$ & $\checkmark$ & $\checkmark$ & - \\
\hline [58] & $\mathrm{X}$ & - & - & - & $\mathrm{X}$ & $\mathrm{X}$ & $\mathrm{X}$ & - & $\checkmark$ & - \\
\hline [59] & $\mathrm{X}$ & - & $\checkmark$ & $\mathrm{X}$ & $\checkmark$ & $\checkmark$ & $\mathrm{X}$ & $\mathrm{X}$ & $\checkmark$ & $\mathrm{X}$ \\
\hline [61] & $\mathrm{X}$ & - & $\checkmark$ & $\mathrm{X}$ & $\checkmark$ & $\mathrm{X}$ & $\mathrm{X}$ & $\checkmark$ & $\checkmark$ & $\mathrm{X}$ \\
\hline [62] & $\mathrm{X}$ & $\checkmark$ & $\checkmark$ & $\checkmark$ & - & $\checkmark$ & $\checkmark$ & - & - & - \\
\hline [63] & $\mathrm{X}$ & - & $\checkmark$ & $\mathrm{X}$ & $\checkmark$ & $\checkmark$ & $\mathrm{X}$ & $\mathrm{X}$ & - & $\mathrm{X}$ \\
\hline [65] & - & - & $\checkmark$ & $\mathrm{X}$ & $\mathrm{X}$ & $\mathrm{X}$ & $\checkmark$ & $\checkmark$ & $\checkmark$ & - \\
\hline [67] & $\mathrm{X}$ & $\checkmark$ & $\checkmark$ & $\checkmark$ & $\checkmark$ & $\checkmark$ & $\checkmark$ & $\checkmark$ & $\checkmark$ & $\mathrm{X}$ \\
\hline [68] & - & - & $\checkmark$ & - & $\checkmark$ & $\mathrm{X}$ & - & - & $\checkmark$ & $\checkmark$ \\
\hline [36] & $\mathrm{X}$ & $\mathrm{X}$ & $\checkmark$ & $\mathrm{X}$ & $\checkmark$ & $\checkmark$ & $\mathrm{X}$ & $\mathrm{X}$ & $\checkmark$ & - \\
\hline [73] & $\mathrm{X}$ & $\checkmark$ & $\checkmark$ & $\mathrm{X}$ & $\checkmark$ & $\checkmark$ & - & $\mathrm{X}$ & $\checkmark$ & $\mathrm{X}$ \\
\hline [74] & - & - & $\checkmark$ & $\mathrm{X}$ & $\mathrm{X}$ & $\checkmark$ & $\mathrm{X}$ & $\mathrm{X}$ & $\checkmark$ & - \\
\hline [75] & $\mathrm{X}$ & $\checkmark$ & $\checkmark$ & $\mathrm{X}$ & $\checkmark$ & $\mathrm{X}$ & $\checkmark$ & $\checkmark$ & $\checkmark$ & $\checkmark$ \\
\hline [77] & $\mathrm{X}$ & $\checkmark$ & $\checkmark$ & $\mathrm{X}$ & $\checkmark$ & $\checkmark$ & $\mathrm{X}$ & $\mathrm{X}$ & $\checkmark$ & $\checkmark$ \\
\hline [79] & $\mathrm{X}$ & - & - & $\checkmark$ & $\checkmark$ & $\checkmark$ & $\mathrm{X}$ & $\mathrm{X}$ & $\checkmark$ & - \\
\hline [85] & $\mathrm{X}$ & - & $\checkmark$ & $\checkmark$ & $\checkmark$ & $\checkmark$ & - & - & $\checkmark$ & - \\
\hline [89] & $\checkmark$ & - & $\checkmark$ & $\mathrm{X}$ & $\mathrm{X}$ & $\checkmark$ & $\checkmark$ & $\checkmark$ & $\checkmark$ & $\mathrm{X}$ \\
\hline [91] & $\mathrm{X}$ & $\mathrm{X}$ & $\mathrm{X}$ & $\mathrm{X}$ & $\mathrm{X}$ & $\checkmark$ & $\mathrm{X}$ & - & $\mathrm{X}$ & $\mathrm{X}$ \\
\hline [94] & - & - & $\checkmark$ & $\mathrm{X}$ & $\checkmark$ & $\checkmark$ & - & - & $\checkmark$ & - \\
\hline [97] & $\checkmark$ & - & $\checkmark$ & - & $\checkmark$ & $\checkmark$ & $\mathrm{X}$ & - & $\checkmark$ & - \\
\hline
\end{tabular}

scale USNs. In this scheme, by taking benefits of the inherent temporal correlation of the mobility of objects in underwater, anchor sensor nodes conduct linear prediction. Every ordinary node guesses their position by using the spatial correlation of object mobility pattern in underwater and weighted-averaging its received motilities from the other sensor nodes. Simulation results of the new scheme show that SLMP can highly minimize the cost of communication while keeping constant relatively high accuracy and coverage in localization. The authors also estimated the impact of different design parameters, such as prediction step, confidence threshold, prediction window, and prediction error threshold, on the performance of localization.
A comparison of various localization techniques is studied above and some in the next coming section which is also shown in Table 2. Localization techniques are compared based on sensor node mobility, range estimation, time synchronization, localization accuracy, network lifetime, link quality, etc. The localized nodes must be time-synchronized if ToA is applied for the range estimation. Localization techniques such as silent positioning are useful in reducing the communication overheads because nodes only receive information and do not transmit any information for localization. As compared to the receiving side, transmission utilizes more energy. Recursive localization is more beneficial to increase coverage. Only for routing protocol, if the localization of 


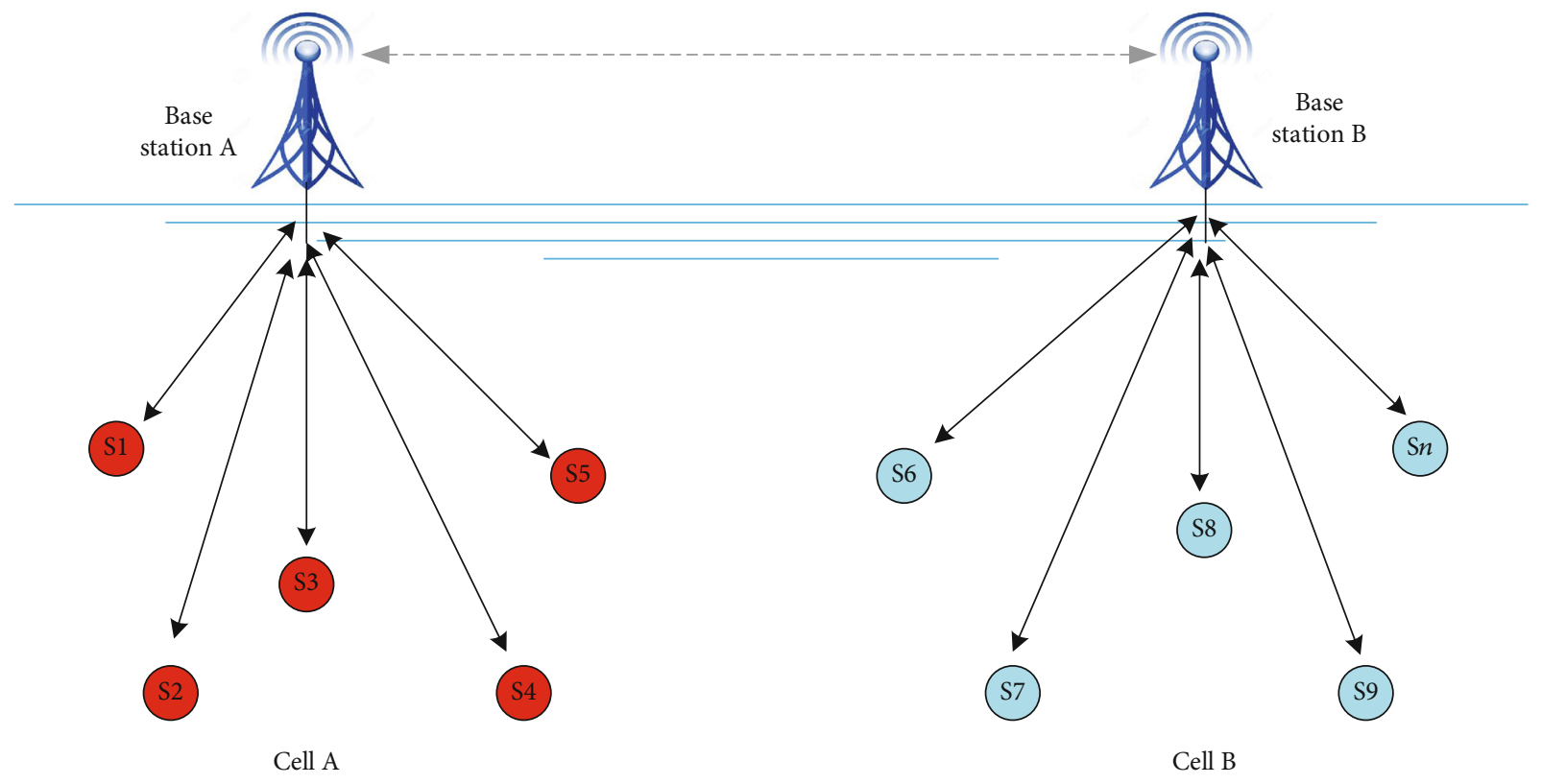

FIgURE 4: Centralized network topology.

sensor nodes is required, then any of the range-free techniques can be applied. Any localization technique can be applied according to the requirement of the application. Also, a performance-based comparison of LSL, PL, and DNR localization techniques is presented in [37]. However, these schemes encounter localization shortcomings. The aforementioned schemes provide a brief description of localization and still many important aspects need to be considered in underwater localization. Therefore, this review can provide a detail explanation of all localization techniques, the existing challenges which face underwater localization, and the future direction for the solution of these challenges.

\section{Techniques Used for UASN Localizations}

Briefly, localization of GPS-based algorithms has been proposed for terrestrial WSNs that, for reasons such as highfrequency GPS, cannot be applied directly to UASNs that attenuate underwater quickly and cannot reach the sensor nodes several meters below the water surface. Also, GPSless systems of localization implement high overhead communication [38, 39]. For the above reasons, it is not possible to directly apply WSN localization to UASNs. In [40], the author's presented the adaptations of the "stochastic proximity embedding algorithm" and "multidimensional scaling algorithm" for UASN. These algorithms conduct concurrent localization of all target nodes compared to the straightforward localization algorithm based on ray tracing that conducts sequential localization of each target node. The proposed methods of localization take into account the bending of acoustic rays and thus, when deployed in real UWSN, give good accuracy. The algorithms have been adjusted to offer directly absolute locations. There are many localization techniques; some of them are categorized as centralized and distributed methods of localization. In centralized localization techniques, firstly, estimate the position of every sensor node in a control center or sink and the sensor nodes do not know their location unless the sink or reference node explicitly sends this information to the node. In this technique, the sensor nodes may be localized at the end of the process such as in the postprocessing phase, or they may gather data periodically for the tracking of sensor nodes. The distributed localization technique allows each sensor node to do localization individually; they are free to do individually localization independently. Centralized and distributed localization techniques are divided into subcategories, i.e., estimation- and prediction-based localization. In the estimation-based localization, the latest available information is used to obtain the current location of the sensor node. For the prediction-based localization, the previous node location, distance information, and anchor location are used to predict the sensor node located at the next moment. Therefore, it is appropriate for mobile UWSN or a hybrid UWSN.

5.1. Centralized Localization (CL). In this technique, first, it calculates the position of each sensor node in the control center or sinks, and the sensor node initially does not know its location unless the sink sends this information explicitly. In this method, nodes may be located at the end of any operation, i.e., postprocessing phase or data may be collected sporadically for sensor node monitoring. In centralized algorithms in [41], in which a key organization (e.g., the control center) exists that gathers all the needed data or estimation (e.g., estimated distances between the nodes in communication ranges and calculates distances to the anchor sensor nodes) and centralizes the sites for the sensor nodes. After the central organization determines the location of the sensor nodes, it sends the location information back to the corresponding sensor nodes. Sensor nodes interact in a centralized algorithm through a base station that includes one cell as shown in Figure 4. 
5.2. Estimation-Based Localization. Estimation-based localization techniques are further divided into subparts by the following:

(1) Hyperbola-Based Localization (HL). Using the localization method based on hyperbola, the location of a sound source, i.e., a target such as mammals, can be identified in the oceanographic scheme using hydrophones such as sensor nodes with known locations [42]. HL also adapts standard oceanographic sound source localization issues to standing 2D UASN localization. In HL, the sensor node sends widerange signals to the anchor node about $1 \mathrm{~km}$ and a centralized sensor node estimates its location. Referring to [43], the authors present a new approach to a better localization accuracy for UASNs. This system uses the hyperbola-based strategy to detect event place and a normal distribution to estimate and standardize error modeling. This efficiency of the method demonstrates a separate enhancement over the estimation of the place of the frequently used minimum squares based on the circle. In terrestrial applications, a multi-iteration and least square measurement system are often implemented to find a decent estimate [44]. But this system is not useful in underwater applications due to its heavy communication costs. At the same moment, it was noted that distance measurement errors often follow a certain shape that can be implemented to further improve the precision of the localization. Authors evaluate and use distributions of measurement errors to improve the precision of localization

(2) Motion Aware Self-Localization (MASL). Due to the long delay in the propagation of signals in the underwater environment, it may take a longer time to collect the number of distance estimates which is required for localization, thus increasing the possibility of obsolete information. MASL's primary objective is to discover the faults in the estimates of distance and provide a precise scheme of localization. The underwater sensor node collects range estimates between the sensor nodes of the neighbors and itself in the MASL method. The distance estimation is performed through certain iterations performance. At each iteration, the algorithm polishes location distribution by distributing the field of an event into smaller grids operation and selecting the area in which nodes reside. In [45], the author models the ocean current as layers of equal density, variable velocity, and the nodes of the sensor move with those underwater currents

(3) 3D Multipower Area Localization Scheme (3DMALS). 3D-MALS varies from the MASL method, which combines the thinking of anchor nodes [46] with a variable rate of transmission energy and the thinking of anchor nodes with vertical mobility of buoys house mechanical device [47] that operates as an elevator for underwater transceivers called
Detachable Elevator Transceiver (DET). DET broadcasts its set of GPS-driven coordinates at different concentrations of energy and then goes down underwater. The unlocalized sensor nodes can retrieve the position of portable anchor nodes and their, respectively, smallest energy concentrations and then send them to the reference or sink node. The reference node can understand the position of each sensor node after gathering data

(4) Area-Based Localization Scheme (ALS). It is a type of scheme that provides an estimate of where the sensor node is in the area of the sensor instead of the precise set of coordinates. Anchor nodes in ALS divide the operating region by sending messages to nonoverlapping areas at different energy levels. ALS is appropriate for the setting where there is no need for accurate location data and when the anchor nodes can change their level of transmission power. The benefit of ALS is that the received signal strength (RSS) is jointly light, range-free, and no synchronization requirement. For applications demanding internet location estimation, ALS is not appropriate, so it is not appropriate for precise localization. In [37], ALS is an algorithm for USNs that is range-free, centralized, and coarse-grained. A sensor node underwater maintains a list of anchor nodes and associated energy concentrations. This data is sent to the reference or sink by the sensor node and the sink discovers the region in which the sensor nodes reside. ALS offers a coarsegrained localization evaluation and it is a centralized localization. Therefore, it is not suitable for largescale USNs and applications that require accurate location estimation. After offering all the regions (one for each anchor node) in which it resides, a primary server offers the sensor node position assessment [48]. On the other hand, USP is a 3D localization algorithm with internal position graininess compared to ALS (i.e., it measures the position of a node within a coordinate system as protecting a location within a subarea). In [49], the authors suggest that only a supper sensor node transmits information from its neighboring sensor nodes and is then shared with nearby sensor nodes. Using this protocol, the packet collision in the network is obviated during node discovery. Also, only seed nodes can make additional results; the remaining nodes in the network do not include power consumption to transmit text messages to their other neighbor nodes. Farthest/Farthest algorithm, Farthest/Nearest algorithm, Nearest/Farthest algorithm, and Nearest/Nearest algorithm are the algorithms used to select additional seed nodes [50]

\subsection{Prediction-Based Localizations}

(1) Collaborative Localization (CL). A Collaborative Localization (CL) scheme [51] consideration of portable UASN applications is where underwater sensor nodes are accountable for gathering ocean depth 


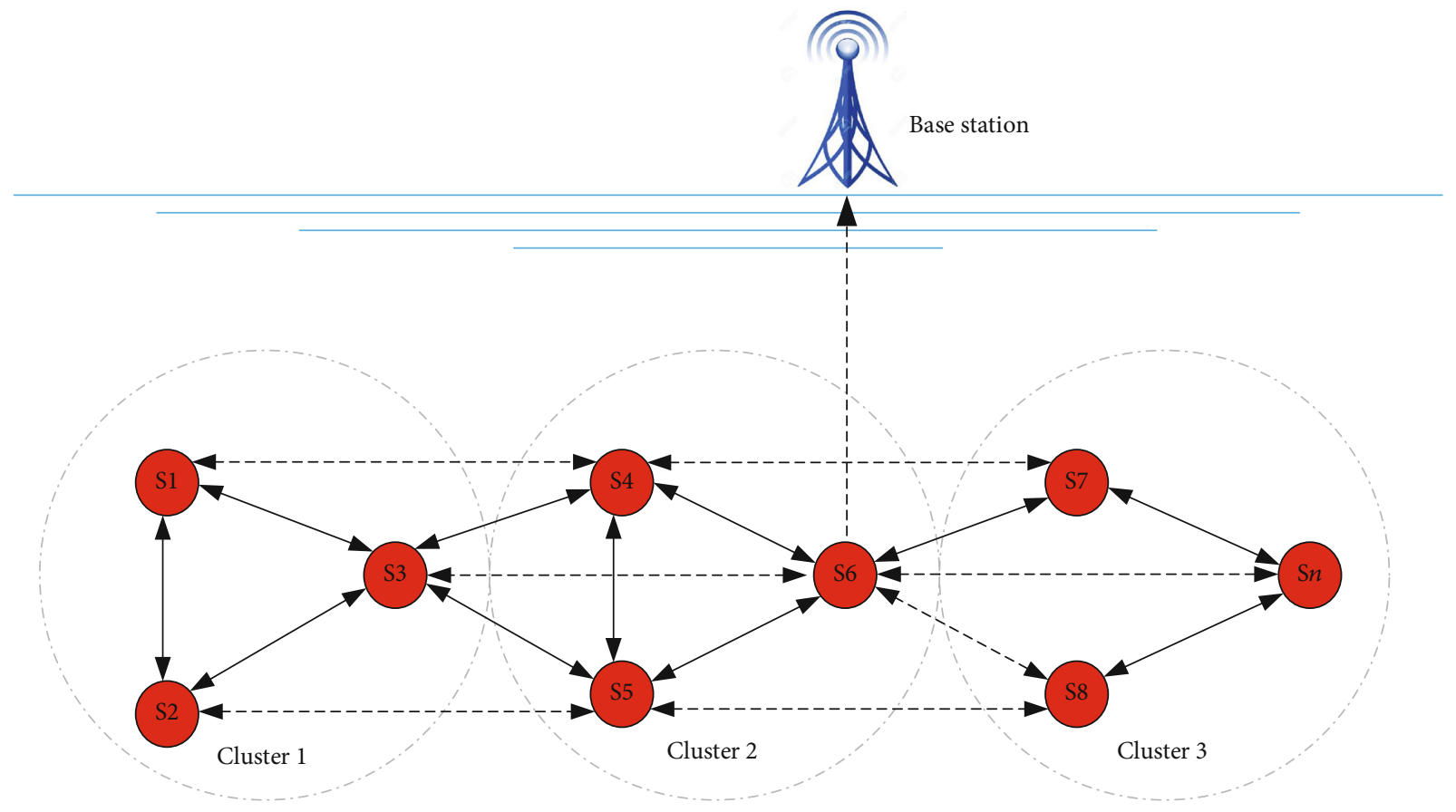

FIGURE 5: Distributed network topology.

information and are accountable for transferring it to the water surface. This architecture uses profilers and supporters of two kinds of underwater sensor nodes. These two kinds of sensor nodes come down underwater, but profilers come down ahead of them, i.e., deeper than other nodes. The distance between profilers and followers is periodically evaluated using the ToA to place the profilers to followers. In particular, the CL algorithm weighed time synchronization algorithm with elevated transmitting delay and a multipath acoustic propagation channel for UASN. By using the new time synchronization protocol, the target position is evaluated using maximum likelihood (MLL) techniques based on the distance of arrival (DoA), since the statistical model between the real and measuring location is based on the marine channel signal envelope. The suggested algorithm also follows the distributed-centralized computing operation that minimizes the energy transmitting node in USNs

(2) Distributed Localization. The distribution localization method allows each sensor node to locate separately or nodes are free to locate such as neighborhood distance, anchor position, and connectivity data and then send all these data separately to the reference or super node. On the other hand, instead of being placed in one central entity, the function of location finding is distributed to the sensors themselves in the distributed localization algorithm. In a distributed network, sensor nodes communicate through peer-to-peer (P2P) as shown in Figure 5.

Distributed positioning algorithms usually assume that anchor sensor nodes are randomly distributed throughout the sensor network, and the percentage of anchor nodes in the network is also high (5\%-20\%). Deploying anchor nodes in terrestrial sensor networks is not a challenge because a GPS-equipped node can act as an anchor node [52]. However, in the case of an underwater field, the network establishes the backbone of the randomly distributed anchor such as ABC nodes; the exact location of which is known before is not a trivial issue

\subsection{Estimation-Based Localizations}

(1) AUV-Aided Localization (AAL). An AAL-based approach is presented in [53] for a hybrid 3D UASN with stationary underwater sensor nodes and AUV traveling in the UASN sector. Using the deadreckoning method, the AUV can get its position underwater. Dead reckoning with the costly inertial piloting machinery is feasible and the position has been periodically calibrated. The AUV goes to the water surface for this purpose at certain periods to achieve GPS coordination from a satellite. A wakeup message can be broadcasted from a separate point on its moving path during the AUV operation cycle. It occurs when AUV receives this signal from the underwater sensor node, it begins the localization action by transmitting a request signal to the AUV, and AUV responds with a reply signal. The pair of requests and response packets provide a two-way algorithm and the response packet includes the AUV coordinates so that the underwater node uses the lateration process to measure its selflocation after the exchange message from three different noncoplanar AUV positions (see Figure 6). 


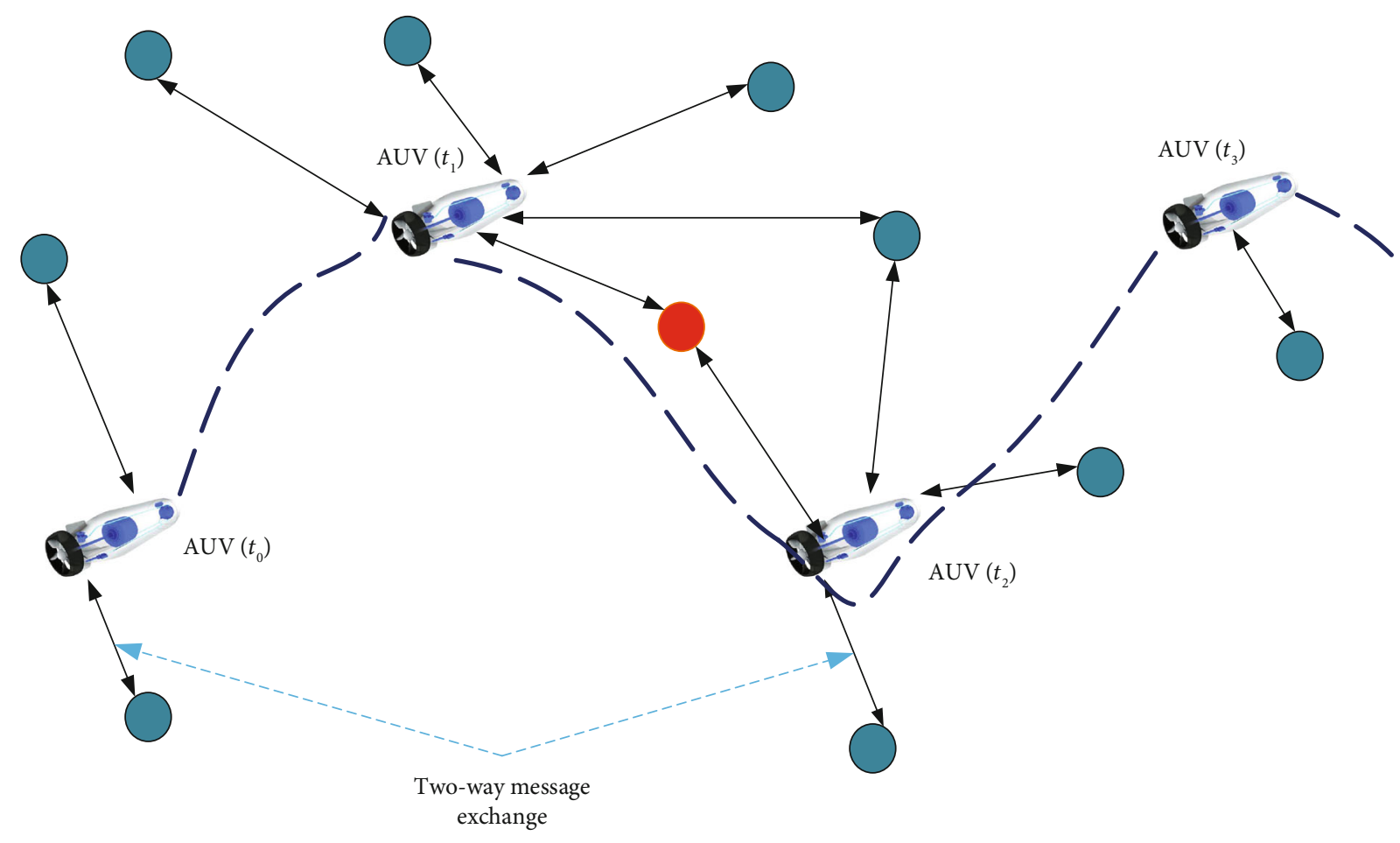

FIgURE 6: AUV cycle of operation with two-way message exchange.

AAL utilizes a two-way display to alleviate the need for synchronization, but on the other side, a sensor node can invest in a silent algorithm more energy than it does and also boosts the protocol's overhead communication. AAL's precision is also influenced by the AUV's localization calibration frequency. An assessment of an AUV's single beacon localization problem modeled as a double integrator [54], where its input is the acceleration in an inertial reference frame and its output is its range to a static beacon. The nonlinear map between range and position enables the range-based observability problem to be considered nonlinear. Two complementary problems are discussed here in the observability evaluation: firstly, the local weak observability of the nonlinear system and secondly, the worldwide observability of a linear time-varying system representation obtained by a worldwide technique of increase

AUVs emit an omnidirectional beacon [55]. When AUV crosses the sensor node at $t_{1}$, this node receives a signal that can be used for the distance calculation $d_{1}$ between the AUV and the sensor node. Similarly, the distance $d_{2}$ can be calculated using the ToA technique as shown in Figure 7. To get the sensor node's coordinate, it requires the AUV's coordinates at two distinct time instants. The location of the node is chosen based on the easy triangulation operation. Also, AUV routing is very complex to guarantee that two necessary beacon messages can be acquired from AUV for each node. Differences in time indicate to some extent the distance. The method is based primarily on the time

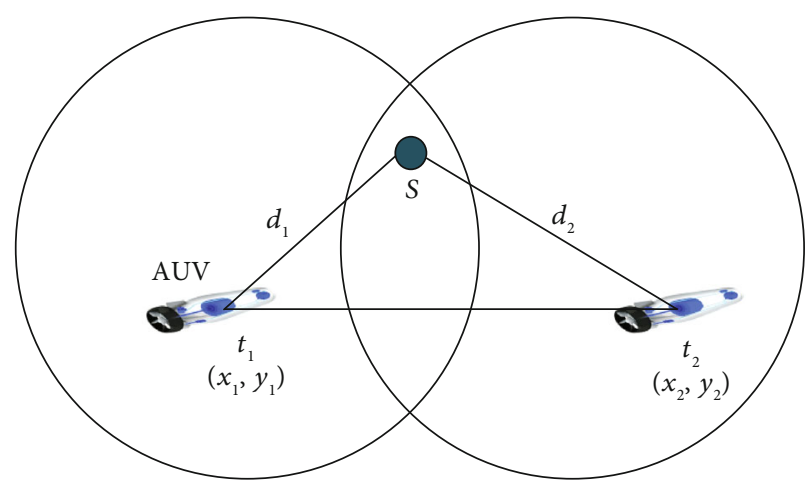

FIGURE 7: Omnidirectional AUV-aided localization.

difference [56]. At distinct locations, AUV broadcasts its coordinates. When receiving messages from more than three noncollinear AUV locations, the underwater nodes estimate their place laterally. This technique has a large delay in localization due to the slow velocity of AUV, which is why it is more useful for stationary USNs than dynamic USNs.

(2) Silent Localization (SL). There are three kinds of messages in AUV-assisted localization systems: wake-up, demand, and reaction messages. The process of positioning consists of three steps [57]. The AUV sends a wake-up signal when it joins the sensing operating region. All sensor nodes will send a request signal or packet after getting the wake-up signal

The AUV then responds with a response packet that contains the coordinates of AUV. For this stage, each 


0 bits 32 bits 64 bits
\begin{tabular}{|c|c|c|c|}
\hline$X$-coordinate & $Y$-coordinate & \multicolumn{2}{|c|}{$Z$ Z-coordinate } \\
\hline \multicolumn{2}{|c|}{ Time-stamp } & MOD & HC \\
\hline
\end{tabular}

FIGURE 8: Localization packet format for proxy localization.

detector node shall communicate with the AUV at least once. Therefore, it is familiar to consume extra energy for localization. Due to the energy consumption of communication between the sensor nodes and the AUV, a better alternative is that during the localization era, only beacons are received by the sensor nodes without interacting with others. This sort of strategy is called a technique of "silent localization." Beacons are interchanged between the sensor nodes and the AUV in the past methods. Lastly, silent localization can considerably decrease underwater localization power consumption.

(3) Dive and Rise Localization (DNRL). Refer to [58], to better define DNRL. It is a distributed, estimationbased localization protocol that applies mobile anchor using the localization of underwater sensor nodes, and these anchor nodes are named as beacons called "Dive'N'Rise (DNR)." By using the same hydraulic laws as the profiling floats laws and regulations, DNR can descend and ascend. When DNR reaches the water's surface, it utilizes GPS receivers and reaches its GPS coordinates while floating on the water's surface. After that dive again, they broadcast their coordinates at several periods until a precalibrated underwater depth. Mobile anchors climb up to the water surface in the first round of localization to obtain the updated coordinates from GPS. They descend and dive or ascend periodically on the second-round journey and so on until the end of the UASN phase. Underwater sensor nodes listen to time-stamped DNR texts and use a ToA method to evaluate distances to DNR beacons using one-way ranges. The range estimates and the coordinates of the anchor node are used in lateration. One advantage of DNRL is the silence that results in low overhead communication and very high energy efficiency. DNR has a wide coverage and gives an accurate estimate as the mobile anchors dive into the vicinity of the underwater nodes and periodically update their position when they reach the surface of the water. On the other hand, for high localization effectiveness, DNRL needed a big quantity of DNR beacons, while DNR beacons are expected to be more expensive than other underwater sensor nodes due to their movement ability

(4) Proxy Localization (PL). PL utilizes the DNRL method to locate the top of the network location. The DNR beacons sink to half of the 3D USN depth. Localized nodes then become proxies of location for those nodes that float at greater concentrations. Location proxies advertise self-coordinates for further localization in proxy place. Nonlocalized underwater nodes can later use and locate themselves with the proxy coordinates. A nonlocalized underwater sensor node picks the trusted proxies between nodes using the hop count metric. Hop count is the distance from a proxy node to a beacon. Error accumulates in iterative methods of the localization at the proxy nodes remote from the beacons. Therefore, proxy nodes with the lowest hop range can be selected to enhance the accuracy of lateration equations as shown in Figure 8

(5) Localization Using Directional Beacons (LDB). LDB is suggested for a 3D UASN hybrid in which stationary underwater sensor nodes are located by AUV like to AAL [59]. When AUV reaches the water's surface, it gets its coordinates from the GPS, dives again to a certain depth, and performs deadreckoning for underwater self-localization. LDB differs from AAL during the localization operation in a way that the AUV travels above the operation area as shown in Figures 9 and 10. It utilizes a directional acoustic transceiver to transmit its position and the transceiver angle. The sensor node uses angle data to map the AUV coordinates with itself to the same horizontal plane. After two or more beacons nodes are obtained, the nodes can assess the location information. The sensor nodes listening to the beacon nodes and the beam form distinct circles $h$. The center of the circle is $(x, y, h)$. Thus, the circle radius can be expressed as follows:

$$
r=\tan \frac{\alpha}{2} \times \Delta h
$$

where $\alpha$ is the angle of conic beacon and $\Delta h=$ $\left|h_{a}-h\right|$. The rough position by using the receiving beacons $(x, y, h)$ can be estimated [60].

On the other side, LDB is a range-free, silent localization method that is more energy-effective than AAL's method. LDB has one disadvantage that the AUV is restricted to traveling above the UASN area, which may be impossible in a real situation. Furthermore, owing to hitting with each other, the frequency of the AUV messages affects the precision of the localization process

(6) Multistage Localization (MSL). To the best of our understanding, the authors suggest the MSL system that defines the DNRL-related by adding coverage and delay an additional localization stage and using effectively located underwater nodes as anchor nodes. An unlocalized node utilizes the coordinates and range estimation from three noncoplanar sensor nodes that can be DNR beacons or a localized sensor node of the underwater sensor. Due to the iterative localization method, MSL has the disadvantage of 


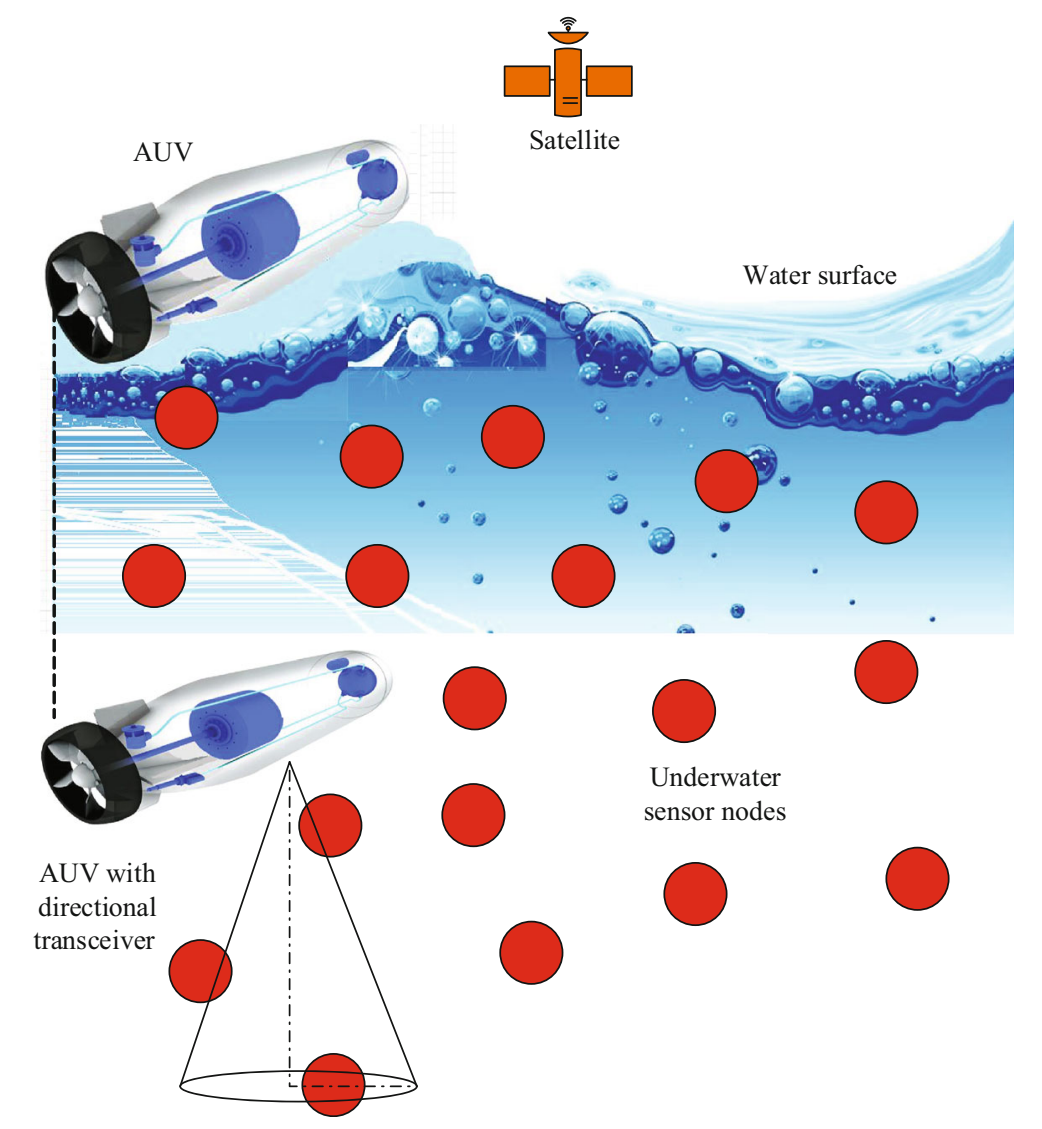

FIgURE 9: AUV with directional beam in the LDB scheme.

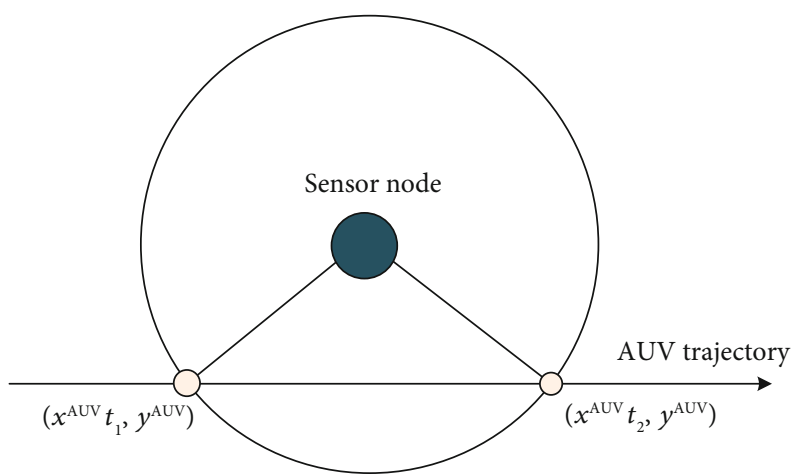

FIgURE 10: Sensor's localization in LDB.

its high overhead communication. For this purpose, therefore, MSL is less energy-efficient than DNRL. Furthermore, localized underwater nodes in MSL provide their estimated locations that already include failures in the estimate. Error accumulates at nodes using coordinates of localized underwater nodes instead of coordinates of anchor nodes. Because of its one-way ToA algorithm, comparable to the DNRL algorithm, MSL also required time synchronization

(7) Underwater Positioning System (UPS). UPS is a system used for monitoring and piloting underwater vehicles or divers through measurements of acoustic range and/or direction, and subsequent triangula- tion of position. UPS is frequently used underwater, including oil and gas exploration, sea science, rescue activities, marine, law enforcement, and military purposes. The authors suggest UPS, an extension of the terrestrial WSN localization system introduced in [61]. UPS is a localization system based on TDoA for standing UASNs. It utilizes four anchors that transfer beacon messages sequentially. One anchor node works as a master anchor and initializes the procedure of localization. Assume the master anchor is selected as the " $\mathrm{A}$ " anchor (see Figure 11). This signal is heard by anchor " $B$ " and sensor node " $\mathrm{S}$ " when the beacon signal is sent. Anchor " $B$ " reacts to anchor " $A$ " by recording the time difference between anchor " $A$ " beacon arrival and the beacon signal transmission time. The anchors " $C$ " and " $D$ " repeat the same successive cycle after the "B" anchor. Node "S" hears these beacons of anchor and calculates the TDoA of beacons. Then, by multiplying them with sound velocity, it transforms TDoA values to range distinctions. Node $S$ is presumed to understand the locations of the anchors and measure self-location using anchor position and trilateration equations range distinctions. Since UPS utilizes TDoA, synchronization is not necessary. The writers also suggest the UPS, i.e., a silent acoustic positioning system for the underwater vehicles/sensors, which depends on the 


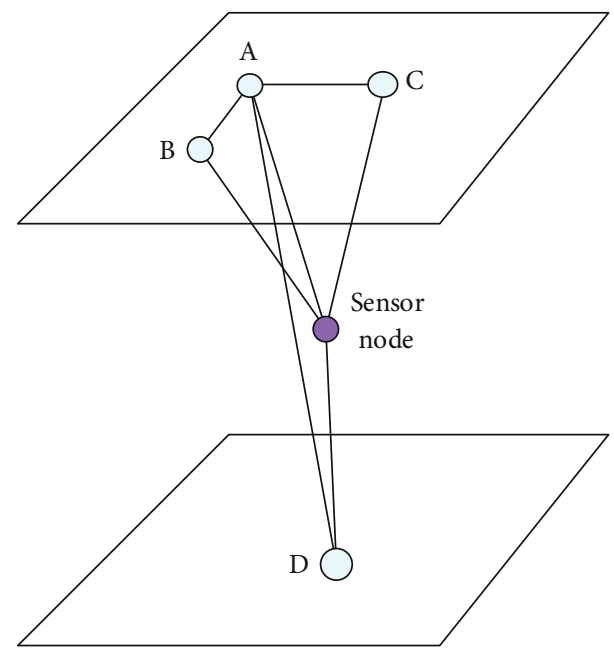

FIGURE 11: Underwater positioning system (UPS) using four anchors.

ToA of RF signals for location estimation from three anchor nodes. UPS comprises essentially two steps: in the first step, from four anchor nodes, they can detect variations in signal arrival times. These time distinctions are converted from the underwater vehicle/sensor to the anchor nodes into range distinctions. In the second step, the transformation of these distance estimates into their coordinates takes place through trilateration. The authors suggested that UPS silent placement should be further emphasized. First, because sensors/vehicles do not transmit any beacons for positioning purposes, it can considerably retain bandwidth and therefore modify network throughput. Second, UPS also applies four or more anchor nodes to an asymmetric UASNs where the underwater vehicles or sensor transmission could not reach. Third, silent positioning offers powerful privacy of location that can assist safeguard sensors/vehicles from detection in critical circumstances

(8) Wide Coverage Positioning (WPS). The UPS algorithm may not uniquely locate all sensor nodes in the four anchor nodes operating region [62]. It indicates that the sensor nodes near the anchor nodes need five anchors to fix the issue. In the event of WPS, four anchors are used whenever distinctive location can be achieved utilizing four anchors called UPS (4); otherwise, WPS will use five anchors (UPS (5)). UPS (4) and UPS (5) are used together to solve the overhead and price of communication for the sensor nodes with four anchors that can already be located. These nodes consume the same number of energy as the initial system of localization

(9) Underwater Sensor Positioning (USP). Using USP, for underwater localization, underwater nodes are fictitious to equip with the help of pressure sensor nodes by which nodes find their depth position. The depth data is used by an underwater node to map the accessible anchors on a horizontal plane on which it rests. While mapping from $3 \mathrm{D}$ to $2 \mathrm{D}$, some of the anchor nodes maybe reside in overlapping locations. In these situations, an underwater node picks out another set of the anchor's sensor node. Localized underwater nodes transmit their location data at each iteration of USP and burn their position estimates based on getting messages from the nodes of neighbors. The unlocalized nodes attempt to constitute localization using only two anchors nodes; the process is called as bilateration. If the two anchors do not compute a new location, the node will wait until it hears from other neighbors' nodes they are already localized. After a slumber period that is preconfigured timing, the same localization operation is reinitiated. In [63], authors present a positioning system in underwater sensor networks. For this, the writers implemented a weighted Gerchberg-Saxton algorithm to address the multipath acoustic propagation problem of various feasible distance measurements between two nodes. A standard positioning technique finds the range in between two sensor nodes which are based on either on initial arrival or the stronger way, but neither may agree to the immediate acoustic route in underwater. In WGSA, based on the ML rules, it can be used for identification of direct path from the overall existing multipaths by connecting each path with a weighting component; it can be understood in a certain way roughly as the chances of that path existence the immediate path

In the WGSA algorithm, the weighting component and the sensor node location are updated periodically or based on iteration, with each iteration computationally easy. USP [64] is based on sensor network technology, which uses many conventional hydrophone stations and GPS sensor nodes above the water surface. Referring to [65], a new underwater acoustic positioning system uses new terminologies. For the range calculation unit, the distance can be calculated alternately based on the time difference by using the connection between propagation noise loss and propagation distance. It is a very simple procedure for processing, and it can use to measure a long-distance winder medium. For an underwater robot, a system of acoustic positioning underwater is shown in Figure 12.

In [66], the geometric configuration of a surface sensor network will maximize the variety of information associated with the target positioning algorithm underwater in a well-defined sense. Because of the white Gaussian noise, the range estimate is not precise and its discrepancy depends on distance.

The Fisher Information Matrix (FIM) and its determinant maximization are used to evaluate the constellation of sensor nodes providing the target's most precise location. Another approach is to locating and mapping Underwater Robotic Fish (URF), 


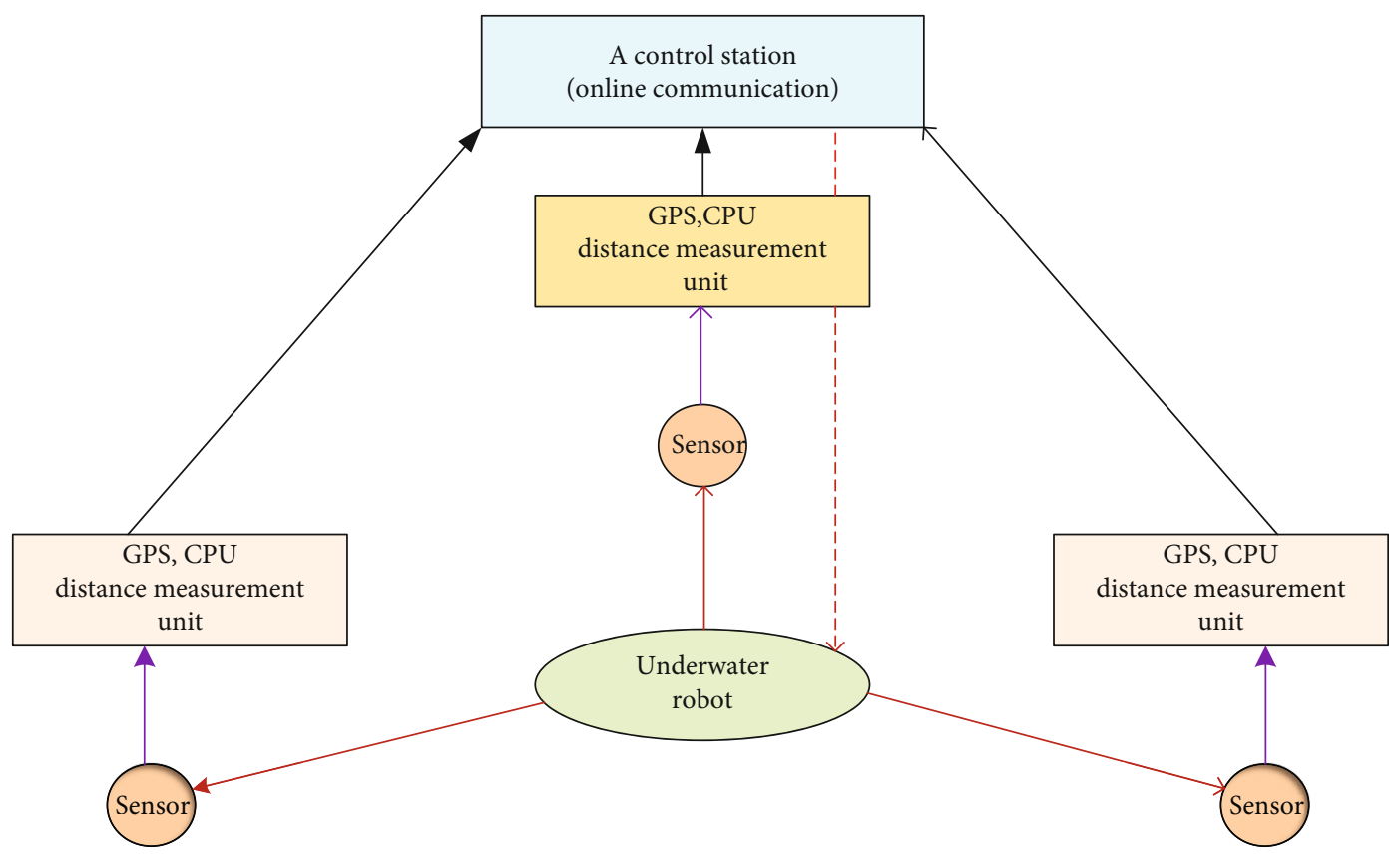

FIGURE 12: Underwater robot-based sensor positioning system.

which is based on both the Particle Filter (PF) system for cooperative localization and the Occupancy Grid Mapping Algorithm (OGMA). The suggested CLPF is more advantageous to use the probabilistic algorithm that they do not have previous data or information about the URF model is needed to accurately attain a 3D localization. If the number of mobile beacon nodes for some traditional localization algorithms is less than four, which is the minimum number, it can achieve good accuracy in the case. To build the environment map, the localization result of CLPF is fed into OGMA. Moreover, even under a normal degree of connectivity, USP has reduced localization achievement than the other surveyed localization methods

(10) Anchor-Free Localization (AFL) Scheme. Conventionally, the existing localization algorithm mostly presumes that the network has a multitude of anchor nodes [67], for the assistant of node positioning. Mostly, it makes the use of AUV or sometimes a sensor node with a particular device, an anchor node, and AFLA. AFLA is especially intended for underwater networks with active restrictions. In the case of AFLA, they do not need anchor node information and use the data of its neighbor's sensor nodes. In [68], the author's present another algorithm for the difficulties in localization that license for node unrelated discovery on the Line-of-Sight (LoS) and any rigid reference sensor nodes. They create a surfacebased reflection anchor-free localization (SBRAL) method in which all nodes use homomorphic deconvolution to establish a reflected communication connection from the water surface. A GPSfree protocol [69] can discover nodes and relative location of nodes. The procedure of node discovery starts with first or an initial seed node with a known position or location. The first seed node can determine the relative positions of neighboring nodes without using inner information and is similar to the first node, other nodes in the network. Selfinitialization includes certain distant nodes becoming seed nodes for further cycles of discovery and so on. Node is originated by seed node $S_{1}$, then $S_{1}$ broadcast the message among its neighbors and receive response from different nodes, and then select a second node $S_{2}$. $S_{2}$ revise the same procedure, broadcast, and receive, then $S_{3}$, and so on. Finally, the sensor nodes in the intersection region of these three seed nodes can determine their position using the trilateration algorithm as shown in Figure 13. Also, owing to the maximum delay in propagation in the underwater medium, the node discovery process may take a long time owing to AFL based on communion range estimates among its neighboring sensor nodes. And the approach to the target station is being viewed as a survivor through the RSSI calibration and RDD without a premeasurement. This algorithm's weakness is fixed by using a moving distance for the PLE assessment instead of the known distance. By using the remaining counter that has a direction of movement until it exceeds, it is an indication to change direction

\subsection{Prediction-Based Scheme}

5.5.1. Scalable Localization with Mobility Prediction (SLMP). SLMP is a method that uses surface buoys, anchor nodes, and common nodes [36]. By using the prior coordinates and their mobility pattern, the anchor node measures its position. As 


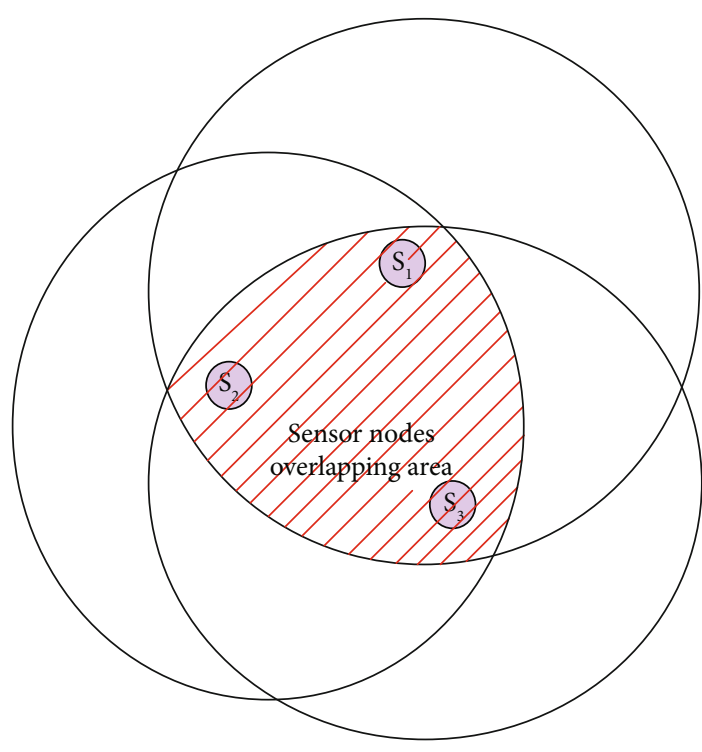

FIGURE 13: AFL using three seed nodes.

the mobility pattern may cease to be used in time, the anchor node periodically checks the pattern's validity. Updates anchor trigger when the model is no longer valid. GPS coordinates are received by the ground buoys and sent to anchor nodes. After predicting its position, an anchor node uses coordinates of surface buoys and lateration measurements of distance to buoys and estimates their position. If the Euclidean distinction is less than a limit between the expected and estimated position, the anchor node will consider its mobility model valid, depending on the mobility pattern, overhead communication, and energy consumption in SLMP. SLMP uses a temporally and spatially connected model of mobility representing the tidal current in shallow waters. Because of this correlated movement, SLMP needed fewer updates, resulting in low overhead interaction and power consumption. Anchor nodes periodically predict their position and through distance measurements check the accuracy of their predictions to surface buoys. They update their mobility pattern if the prediction is incorrect and send a signal to the ordinary sensor nodes. With their mobility model, which is updated when anchor nodes announce an update alert, ordinary sensors predict their location.

The algorithms are further classified into range-based and range-free [70].

\section{Range-Based Algorithm}

Accurate estimation of distance or angle measurement is made in the range-based algorithm, and TDoA, ToA, and AoA are the algorithms used for this purpose. Due to certain constraints like its time-varying characteristics, which are rarely used in UASNs, RSSI is not too convenient. TDoA employs arrival time difference and is the time difference between distinct transmission mediums or beacons from distinct reference nodes used to assess the distance between two objects. Similarly, ToA is the time of arrival using for distance estimation. In the suggested range-based algorithms [71], the most frequently used technique for UASNs is the ToA algo- rithm, and it is favored in UASNs as compared to terrestrial. It is several times smaller than the radio signal in the atmosphere owing to the sound velocity in water. ToA is mostly implemented to UASNs, although synchronization among nodes was needed by ToA. A hybrid bearing and range-based UWSN has been studied in [72]. The authors explore the impact of comparative sensor-target geometry on the underwater target location's prospective results. The optimality criterion function is built as the Fisher Information Matrix (FIM) determinant, and the mean square error (MSE) is also provided with a comparable assessment. The MSE is minimized only if, under the assumption of a set distance between the detectors to the underwater goal, the determinant of the FIM is maximized. The authors suggest a localization method called a Two-Phase Time Synchronization-Free Localization Algorithm (TP-TSFLA) in [73]. TP-TSFLA comprises of two algorithms, the algorithm of range-based assessment and the algorithm of range-free. The writers discuss a time synchronization-free localization system in the first algorithm that is based on the method of Particle Swarm Optimization (PSO) to achieve unknown node coordinates. In the second algorithm, authors present a Circle-Based RangeFree Localization Algorithm (CRFLA) to locate the unlocated nodes that cannot acquire position information through the first phase. For the second phase, finding the location is the conduct of those sensor nodes situated in the first phase such as helping the new anchor nodes. Below is a comprehensive TDoA, ToA, AoA, and RSSI description.

6.1. Time Difference of Arrival (TDoA). Localization is one of the main issues in a network of wireless sensors. The TDoA is a commonly used method for localizing underwater. By using the TDoA method, a goal with anchor nodes can be asynchronous [74]. An asynchronous ToA-based localization method is used [75-77], in which the transmission source time is unknown and ToA measurements have a favorable prejudice due to the synchronization mistake which can lead to a big localization mistake. One method is to use TDoAbased measurement to fix this issue, which does not rely on a transmitter source's transmission time. They also use the method of SDP to turn the nonconvex MLE issue into a convex issue. Since GPS signals are extremely attenuating underwater, it is necessary to develop a precise range-based algorithm for locating underwater. Authors define a sequential method for time synchronization and localization in the acoustic channel [78]. Authors consider it a realistic situation in which nodes are not time-synchronized and underwater sound speed is also unknown, validating the issue of localization as a series of two linear estimation issues. The velocity of propagation that changes with depth, temperature, salinity, etc., anchor nodes and unlocalized (UL) nodes cannot be regarded as time-synchronized, and the nodes of the water current are constantly moving or their self-motion. The authors define a fresh sequential algorithm in an underwater setting for joint time synchronization and location. This associated method is based on exchanging information between the anchor and UN nodes, using the directional navigation algorithm used in nodes to achieve precise short-term 
estimates of movement and using continuous nodes. This method achieves a precise localization environment is utilizing only two anchor sensor nodes and exceeds the benchmark systems when node synchronization and propagation speed data are unknown.

6.2. Time of Arrival (ToA). In ToA-based systems, the target must be symmetric with anchor sensor nodes. The author's proposed a new model [79] called ToA-Based Tracked Synchronization (ToA-TS) expands GPS as localization to outline where beacon signals do not coincide and monitor the submersible while synchronizing time. And the beacon that transmits a signal will include the beacon's location data gathered from GPS, as well as the time stamp that represents the time it was sent based on worldwide time. On the receiver hand, the message's receiving time is saved according to the submersible's local clock. In [80], the investigator introduces a mixed localization algorithm for Time-of-Flight (ToF) and Direction-of-Arrival (DoA) which is desirable in the setting of shallow underwater control applications as well as harbor monitoring operations. Localization of both ToF and DoA can be measured using a single-way range operation. Then, we need to combine ToF and DoA to reduce the number of the reference node. ToF measurements are performed by calculating the transmission propagation time between the moored reference antenna at the bottom of the tank and an antenna array. The DoA measurement is performed by evaluating the signal touching DoA angle on the antenna array. For real-time positioning in [81], a cooperative low overhead monitoring method for portable underwater networks measures vehicle location in real-time circumstances. Where nodes follow more predictable paths, the efficiency of localization can be improved by strategically positioning beacons most importantly, so in this scenario, all cars are likely to hear a beacon periodically. A joint localization and time synchronization solution considers the underwater environment's stratification impact; thus, compensating for the prejudice in the distance calculation induced assuming sound waves travel underwater in straight lines. The precision of both is greatly enhanced to combine time synchronization and localization [82]. The issue of locating an underwater sensor node in $[83,84]$ is investigated by authors based on message broadcasting from various ground nodes. With the ToA readings from a multicarrier modem based on SDP, each sensor node can locate itself to the receiver nodes on the grounds of travel time differences between various sensor nodes.

6.3. Angle of Arrival (AoA). AoA is a distributed localization and orientation algorithm, which assumes that all unknown sensor nodes can detect incident signal angles from nearby sensor nodes $[85,86]$. In this technique of localization, orientation and designing are several hops away from the beacon data. Under the noisy angle estimation assumption, this algorithm is intended. An evident DoA is initialized as the azimuth direction of peak power in the case of a lowresolution transducer. A tiny array is used as an antenna and the phase derivative along the array axis is used as a measure of the obvious AoA's sine. Perhaps the obvious DoA lies outside the precise range of arrivals. The reason can be understood from a situation between two array components with a null attenuation. The AoA capability offers nearby nodes about the node's axis for each node bearing [87]. Radial is an angle from which an object can be seen from another point or a reverse bearing soon. In cases where nodes transmit their bearings concerning beacon nodes, AoA-based systems are accountable. In [88], A scheme used to collect coincident time and AoA information at some $\mathrm{GHz}$ is defined. Also, the algorithm for data processing is described and its outcomes are analyzed from information collected in two distinct structures. A model is suggested based on the measurement consequence used by Saleh and Valenzuela (1987) in the clustered double Poisson ToA model. In the time-angle indoor multipath data, an accurate clustering shape was determined. The information maintains the temporal clustering and the angle clustering shape has also been found. Each cluster's mean angles were determined to be split evenly over all angles.

6.4. Received Signal Strength Indicator (RSSI). There are many methods for measuring signal strength with RSSI such as distance from the nodes and the received signals. The signal power obtained mostly depends on the radio wave propagation path loss impact. If there is an obstacle between the sources of transmitter and receiver, the signal power can fall considerably on the respective obstructed connection, which is degrading the range estimate for precision [89, 90]. The author has suggested an RSS-based localization algorithm based on Maximum Likelihood Estimation (MLE) for obstructed fields with unknown Path Loss Exponent (PLE). An RSS-based localization was implemented in [91] for UWSNs using acoustic signals. They proposed a novel SDP estimator, including an RSS-based technique and a frequency-dependent differential process, called the FDRSS-based approach, based on the UWA transmission loss (TL) model; these two techniques provide important localization effectiveness. For an underwater range estimation based on RSS, a fresh implementation of the Lambert $W$ function is suggested. It demonstrates that the derived mathematical equation can calculate precise distance using four iterations in [92] using the Lambert $W$ function. Authors predict that more UWSN applications, particularly sensor networks, will be found in the Lambert $W$ function. The author provided an RSS-based UASN localization algorithm with stratification compensation (NRA-WSE) in [93]. In NRAWSE, the Urick propagation model brought the RSS-based localization to quality, and the stratification impact is modeled by the use of the ray tracing algorithm, further adopting the Newton-Raphson algorithm for the source localization solution. One of the fundamental problems in UWSNs is to determine the location of the detectors mostly achieved by evaluating the distance between anchor nodes and unknown nodes. Ranging algorithms are generally performed by either assessing the ToA or RSS signal. Previous studies traded underwater with the assumption of straight-line wave propagation with the location of sensors. However, the truth is that, due to the inhomogeneity of the underwater framework, acoustic waves move through a 
curved manner. The writers used an analytical relationship $[94,95]$ that describes the loss in transmission of acoustic waves in the inhomogeneous landscape and then on a relationship basis. A unique algorithm is suggested to estimate the variety between two sensor nodes and show that the underwater source isotropically radiates acoustic noise and uses the RSS, which is drawn from the UWSN's sensors to correctly measure the source node location. Authors describe another sensor model relationship between range, RSS, and the 3D Extended Kalman Filter (EKF) in [96]. $\mathrm{RF}$ sensor is based on the underwater medium linearity of the RSSI value. Based on the respective sensor model, the vehicle measures the loudness of the signal and measures the distance to the beacon.

\section{Range-Free Algorithm}

To use range-free localization algorithms, we do not need to use a variety of bearing information; it only provides a coarse estimation of the node of the sensor position that is distinct from the range-based algorithm. A hybrid localization algorithm with multihop mobile underwater acoustic networks has been suggested by the author in [97] to enhance the effectiveness of the localization scheme in a mobile underwater medium. The sensor nodes in the network are split into multistage nodes for this algorithm and each stage has a distinct localization operation. Both range-based algorithms and range-free algorithms are used to enhance localization precision and decrease communication costs. Moreover, this algorithm does not involve any previous understanding of the velocity of motion that is readily used in an underwater medium. Also, the range-free algorithm is categorized into the hop count-based, area-based algorithm, and centroid algorithms.

7.1. Hop Count-Based Algorithms. The anchor sensor nodes are placed in the hop count-based algorithm along with the boundaries or corners of a square grid. Three algorithms are DV-Hope, solid positioning algorithm, and DHL. The DV-Hope utilizes an average estimation of the spectrum of hope and the counted number of hops to estimate the distance to the anchor node. Robust positioning algorithm is used to raise DV-Hop by inserting an extra refinement step, while DHL may use density consciousness to dynamically rather than statically estimate distance.

For actual deployments where sensor node distribution is more likely in some areas, it is uneven and sparse, such as DHL has been recommended to improve the accuracy of position estimation when the distribution of nodes in the network is not uniform [52]. This program needs taking into account the density of the neighborhood of the node calculates the average hop distance, as well as the wrong facts distance estimates tend to accumulate with increasing path length.

7.2. Area-Based Algorithm. Area-Based Localization Scheme (ALS) and approximate point in a triangle (APIT) are the two area-based algorithms. ALS is a centralized range-free system whose main benefits are the resistance and simplicity in underwater to the variable sound speed. They can measure the location of a sensor node within a specific operating area and the sensor node clock must be synchronized in time. It presents the most recent algorithm based on ALS. For instance, 3D multipower area localization scheme (3DMALS) has the function of extending 2D-ALS to $3 \mathrm{D}$, whereas APIT requires a heterogeneous network. Anchors are fitted with high-power transmitters and can accurately acquire location data using GPS coordinates. In [98], the author's used a new technique which is based on Mel Frequency Cepstral Coefficients (MFCCs). The underwater acoustic method is generally nonlinear and very hard to evaluate, so a correct nonlinear algorithm is required. Thus, MFCC is applied to underwater radiated noise extraction characteristics. MFCC is, therefore, an efficient recognition and extraction algorithm.

7.3. Centroid Algorithm. The centroid algorithm is a localization algorithm based on proximity and coarse-grained range-free. The disadvantage of centroid localization algorithm is due to the high localization error because of the centroid formula, where $\left(X_{e n}, Y_{e n}\right)$ is the estimated location of the receiver.

$$
X_{e n}, Y_{e n}=\left(\frac{X_{1}+X_{2}+X_{3}+\cdots X_{n}}{n}, \frac{Y_{1}+Y_{2}+Y_{3}+\cdots Y_{n}}{n}\right)
$$

For the 3D network application, the centroid algorithm which focuses on node self-localization may not be suitable. A distributed joint establishment control of generic multiagent robots [99] is capable of underwater medium applications such as multiautonomous surface vehicles. The ASV agent's goal is to keep some institutions in a predefined geometric shape, particularly the formation of symmetric structures. Furthermore, the centroid of this to-be-sustained establishment is to come after a denominated leader agent whose dynamics appear like that of its other followers. A fresh 3D underwater localization algorithm utilizes three floating buoys on the ground called anchor nodes that are fixed with GPS, RF, and acoustic transceivers. A high number of nodes underwater sensors are installed at distinct depths. These can be anchored to the bottom of the ocean and fitted on the surface of the water with floating buoys. These sensor nodes thus have restricted capacity for movement and are referred to as semistationary nodes.

\section{Performance Evaluation of Underwater Localization Schemes}

In previous sections, we discussed comprehensively the underwater localization schemes and underwater acoustic networks. The underwater localization schemes are analyzed and compared with each other. This section contains the performance evaluation of the abovementioned underwater localization schemes concerning various aspects of localization and underwater communication. The performance evaluation is presented in Table 3. 
TABLE 3: Analysis of UASNs and underwater localization algorithms.

\begin{tabular}{|c|c|c|c|}
\hline $\begin{array}{l}\text { Localization } \\
\text { algorithms }\end{array}$ & Selection methodology & Advantages & Drawbacks/issues \\
\hline CL [41] & Use a control sink. & $\begin{array}{l}\text { Locate nodes at both condition: } \\
\text { postprocessing or at the end. }\end{array}$ & Required centralized center. \\
\hline HL [42] & Apply hydrophones/TDoA. & $\begin{array}{l}\text { Adopt standard oceanographic } \\
\text { localization issues. }\end{array}$ & Range is limited. \\
\hline MAS [45] & Range estimates. & Provide precise localization. & No error estimation is performed. \\
\hline 3D-MASL [46] & Use DET (broadcasting). & Limited energy consumption. & Transmission rate is variable. \\
\hline ALS [46] & Central sink/RSS. & Reduce energy consumption. & Unable to estimate the exact location. \\
\hline CL [51] & Automatic localization. & Reduce localization errors. & Valid only for limited nodes. \\
\hline AAL [53] & ToA/AUV. & Time-synchronized. & Invest more energy. \\
\hline SL [57] & TDoA. & Required no time synchronization. & Channel modeling error is not estimated. \\
\hline DNR [58] & GPS/acoustic. & Reduce communication cost. & Do not consider the sensor mobility. \\
\hline LDB [59] & AUV/3D deployment. & Localization error estimation. & Unable for 3D-free drifting UASN. \\
\hline UPS/TPS [61] & TDoA/extension of TWSN. & Use for oil, gas, and sea exploration. & $\begin{array}{l}\text { Applicable only for outdoor } \\
\text { WSN/not for ToA. }\end{array}$ \\
\hline WPS [62] & $\begin{array}{l}\text { Based on the premise of } \\
\text { synchronized clock. }\end{array}$ & $\begin{array}{l}\text { Low energy consumption and } \\
\text { low localization latency. }\end{array}$ & Work only in a finite region. \\
\hline USP [63] & $\begin{array}{l}\text { Use hydrophone stations } \\
\text { and GPS nodes. }\end{array}$ & $\begin{array}{l}\text { Work in both } 2 \mathrm{D} \text { and } \\
3 \mathrm{D} \text { environments. }\end{array}$ & $\begin{array}{l}\text { Nodes reside in the overlapping } \\
\text { area while mapping from } 2 \mathrm{D} \text { to } 3 \mathrm{D} \text {. }\end{array}$ \\
\hline AFLA [67] & AUV/nodes with a particular device. & No need of anchor nodes. & $\begin{array}{l}\text { Only depend on the neighbor nodes, } \\
\text { no communication with anchors. }\end{array}$ \\
\hline SBRAL [68] & Surface water communication links. & No need for LoS/ToA. & Link quality is not convenient. \\
\hline SLMP [36] & Surface buoys and anchor nodes. & Reduce communication cost. & Not suitable for dynamic environment. \\
\hline ToA [70] & Acoustic/targets must be synchronized. & Most frequently used for UASN. & Time synchronization is required. \\
\hline TDoA [70] & Known transmission time. & $\begin{array}{l}\text { Do not depend on the transmission } \\
\text { time of source. }\end{array}$ & High cost and energy consumption. \\
\hline AoA [70] & Based on the arrival angles. & $\begin{array}{l}\text { All unknown nodes can detect } \\
\text { incident signal angles. }\end{array}$ & Ultrasound receiver increases the cost. \\
\hline RSSI [70] & $\begin{array}{l}\text { Depend on the strength of received } \\
\text { signal and path loss impact. }\end{array}$ & $\begin{array}{l}\text { Applicable in asynchronous } \\
\text { scenarios. }\end{array}$ & Loss caused by multipath fading. \\
\hline
\end{tabular}

\section{Challenges and Open Issues}

UWSNs offer a range of applications from civil, military, and many others. During the localization process, the detected data can only be interpreted usefully when responding to the location of the sensor node, which makes localization a key problem. Thus, GPS receivers are frequently used for obtaining this in terrestrial WSNs; it is infeasible in UWSNs as less propagation of GPS signals in an underwater environment. The most secure method of communication for underwater is acoustic wave communication. Underwater acoustic channels, however, are faces with low high bit error, bandwidth, and high delay in propagation due to severe physical layer circumstances. Observing the current undersea acts, they are often based on complicated suppositions such as time synchronization, as they have exploited the ToA technique for the most part. When AUV reaches the water surface, it gets its coordinates from the GPS; it dives again to a certain depth and performs dead-reckoning for undersea self-localization. The difference between LDB and AAL is that the AUV is traveling above the operating region during the localization procedure and utilizes a directional acoustic transceiver to transmit its position and the angle of the trans- ceiver beam. Synchronization might be a large challenge in such an atmosphere. The challenges of UWSN are divided into two categories: first, the underwater environment, such as the deployment of reference nodes in the deep sea, node mobility, internode time synchronization, and signal reflection owing to barriers and reflective surfaces. The second is the underwater acoustic channel such as long delay in propagation, multipath fading and shadowing, sound speed variation, low bit rate, heavily unreliable and asymmetric SNR, and asymmetric energy consumption. Thus, by increasing energy consumption resultantly decreases network lifetime and efficiency. Here, we presented some basic challenges and open issues that need to be solved in the recent future.

9.1. Time Synchronization. As discussed in Section 5, time synchronization is the main aspect of underwater localization. The surface sensor nodes are time-synchronized by using GPS or DNR, while the underwater sensor nodes cannot be time-synchronized, and the clocks of underwater nodes are subject to skew and offsets [60].

9.2. Reliability. Reliability is a key point in ensuring reliability in all forms, such as hop-by-hop, data, and end-to- 
end reliability. Successfully forwarding and transferring data between participating sensor nodes in the UASN is an important aspect of reliability. Reliability guarantees successful delivery of packets between sensor nodes participating in collaborative processes [100]. The review of this study found that reliability is the most important aspect, but unfortunately, most of the current studies have not considered. Therefore, it is very important to propose a cooperation algorithm that considers this reliability.

9.3. Node Mobility. While it is reasonable to assume that nodes in terrestrial networks remain static, underwater nodes will inevitably drift due to underwater currents, winds, shipping activity, etc. Nodes may drift differently as the oceanic current is spatially dependent. While reference nodes attached to surface buoys can be precisely located through GPS updates, it is difficult to maintain submerged underwater nodes at precise locations. This may affect localization accuracy.

9.4. Efficiency. Efficiency is also a basic aspect in a communication network to provide an efficient cooperative mechanism and to facilitate communication among different nodes. Based on our review of the current algorithms, it has been found that no method takes into account this aspect $[100,101]$. Collaborative controlling activities require an efficient method for successful data forwarding and delivery in underwater localization. It is also required to include efficiency in cooperative game techniques to use resources that ensure efficient delivery of information, if not, then the cost of such information delivery will increase, i.e., delays and throughput.

9.5. Sound Speed Variation. Most of the range-based localization techniques assume constant speed underwater sound, and it is depending on the water pressure, salinity, and temperature. Without measuring the sound speed, the accuracy of distance measurements based on ToA approaches may be degraded [60]. For a fair performance comparison of all techniques, they should be evaluated using a common and accurate sound speed scheme.

9.6. Security and Privacy. Most of the researchers do not consider these two points when designing positioning algorithms; however, there is no doubt that they play a key role in underwater localization. Security attacks for underwater localization and countermeasures, as well as the issue of privacy in underwater localization and countermeasures, are discussed in [102]. The sensor node must display certain information to be localized, which can be lead to privacy holes. Location privacy is discussed in both the locationrelated information collection step and the estimation step. These attacks include DoS, range-based, no range estimation attacks, noncooperation, and false advertising information.

\section{Conclusion}

This paper presents a review of UWSNs, underwater localization, localization techniques, and the existing challenges in the underwater environment. The paper mainly focused on the approaches recently used in underwater localization. Localization for UWSN is an important problem that attracts considerable interest from scientists working on localization underwater. In this paper, the unique characteristics of UWSN and underwater localization are explained in detail. Furthermore, the paper presented the localization basics, localization architecture, and the techniques used for underwater localization. A variety of underwater localization techniques are discussed and compared with each other based on their application and efficiency as shown in Table 2. Also, a range-based and range-free localization algorithm is discussed which included TDoA, ToA, AoA, and RSSI. Finally, the paper presented the existing challenges and issues in underwater localization and underwater acoustic communication. For short, it is not feasible to say that any particular method of localization is the best for all situations because each one has certain strengths and weaknesses and constancy for a particular situation. The ultimate goal of this review is to encourage and promote new scientists in the region by offering a basis on the so far suggested underwater localization. The field of USNs and localization is growing quickly, and yet many difficulties need to be investigated in the future.

\section{Conflicts of Interest}

The authors declare that they have no conflicts of interest.

\section{Acknowledgments}

This research was supported by the National Natural Science Foundation of China under Grant 61801166 and supported in part by the Key Research and Development Program of Jiangsu under Grants BE2017071 and BE2017647. This was also supported by the Fundamental Research Funds for the Central Universities under Grant 2019B22214 and in part by the Basic Science Research Program through the National Research Foundation of Korea (NRF) funded by the Ministry of Education under Grant NRF-2018R1D1A1B07043331.

\section{References}

[1] H.-P. Tan, R. Diamant, W. K. G. Seah, and M. Waldmeyer, "A survey of techniques and challenges in underwater localization," Ocean Engineering, vol. 38, no. 14-15, pp. 1663-1676, 2011.

[2] N. Yashwanth and B. R. Sujatha, "Wireless sensor node localization in underwater environment," in 2016 International Conference on Electrical, Electronics, Communication, Computer and Optimization Techniques (ICEECCOT), Mysuru, India, December 2016

[3] M. Isik and O. Akan, "A three dimensional localization algorithm for underwater acoustic sensor networks," IEEE Transactions on Wireless Communications, vol. 8, no. 9, pp. 44574463, 2009.

[4] D. Park, J. Jung, K. Kwak, W. K. Chung, and J. Kim, “3D underwater localization using EM waves attenuation for UUV docking," in 2017 IEEE Underwater Technology (UT), Busan, South Korea, 2017. 
[5] M. Raja, "Application of cognitive radio and interference cancellation in the L-band based on future air-to-ground communication systems," Digital Communications and Networks, vol. 5, no. 2, pp. 111-120, 2019.

[6] M. M. K. Inamullah, I. Khan, and S. K. Haider, "Compensation of magnetic sensor with electric compass," in 2017 7th IEEE International Symposium on Microwave, Antenna, Propagation, and EMC Technologies (MAPE), Xi'an, China, October 2017.

[7] D. Park, K. Kwak, J. Kim, and W. K. Chung, "3D underwater localization scheme using EM wave attenuation with a depth sensor," in 2016 IEEE International Conference on Robotics and Automation (ICRA), Stockholm, Sweden, May 2016.

[8] C. Kim, S. Lee, and K. Kim, "3D underwater localization with hybrid ranging method for near-sea marine monitoring," in 2011 IFIP 9th International Conference on Embedded and Ubiquitous Computing, Melbourne, VIC, Australia, October 2011.

[9] M. Erol-Kantarci, H. T. Mouftah, and S. Oktug, "A survey of architectures and localization techniques for underwater acoustic sensor networks," IEEE Communications Surveys \& Tutorials, vol. 13, no. 3, pp. 487-502, 2011.

[10] S. Kaivonen and E. C.-H. Ngai, "Real-time air pollution monitoring with sensors on city bus," Digital Communications and Networks, 2019.

[11] J. Heidemann, W. Ye, J. Wills, A. Syed, and Y. Li, "Research challenges and applications for underwater sensor networking," in IEEE Wireless Communications and Networking Conference, 2006. WCNC 2006, Las Vegas, NV, USA, 2006.

[12] M. Goswami and H. M. Kwon, "Submillimeter wave communication versus millimeter wave communication," Digital Communications and Networks, 2019.

[13] I. Ullah, Y. Liu, X. Su, and P. Kim, "Efficient and accurate target localization in underwater environment," IEEE Access, vol. 7, pp. 101415-101426, 2019.

[14] D. Wang, L. Yang, and X. Cheng, "Underwater localization and tracking based on semi-definite programming," in 2013 IEEE International Conference on Signal Processing, Communication and Computing (ICSPCC 2013), KunMing, China, 2013.

[15] H. Yu and W. Li-Hua, "Underwater localization iterative algorithm based on geomagnetic anomaly inversion," in 2012 International Conference on Industrial Control and Electronics Engineering, Xi'an, China, 2012.

[16] M. Erol, L. F. M. Vieira, A. Caruso, F. Paparella, M. Gerla, and S. Oktug, "Multi stage underwater sensor localization using mobile beacons," in 2008 Second International Conference on Sensor Technologies and Applications (sensorcomm 2008), Cap Esterel, France, 2008.

[17] Y. Y. Al-Aboosi and A. Z. Sha'ameri, "Improved underwater signal detection using efficient time-frequency de-noising technique and pre-whitening filter," Applied Acoustics, vol. 123, pp. 93-106, 2017.

[18] P. Carroll, K. Domrese, H. Zhou, S. Zhou, and P. Willett, "Localization of mobile nodes in an underwater distributed antenna system," in Proceedings of the International Conference on Underwater Networks \%Systems - WUWNET '14, Rome, Italy, 2014.

[19] M. M. K. Inam Ullah, S. H. E. N. G. Gao Ming, and Z. Khan, "A survey on underwater localization, localization techniques and its algorithms," in Proceedings of the 3rd Annual Interna- tional Conference on Electronics, Electrical Engineering and Information Science (EEEIS 2017), 2017.

[20] T. Rossby, D. Dorson, and J. Fontaine, "The RAFOS system," Journal of Atmospheric and Oceanic Technology, vol. 3, no. 4, pp. 672-679, 1986.

[21] A. Turetta, G. Casalino, E. Simetti, A. Sperinde, and S. Torelli, "Analysis of the accuracy of a LBL-based underwater localization procedure," in 2014 Oceans - St. John's, St. John's, NL, Canada, 2014.

[22] M. Jouhari, K. Ibrahimi, H. Tembine, and J. Ben-Othman, "Underwater wireless sensor networks: a survey on enabling technologies, localization protocols, and internet of underwater things," IEEE Access, vol. 7, pp. 9687996899, 2019.

[23] Y. Tanaka, Y. Nishida, J. Ahn, and K. Ishii, "Underwater vehicle localization considering the effects of its oscillation," in 2019 IEEE Underwater Technology (UT), Kaohsiung, Taiwan, Taiwan, 2019.

[24] X.-y. Sun, L. Nan-song, and X. Liu, "Three-dimensional passive localization method for underwater target using regular triangular array," in 2019 13th Symposium on Piezoelectrcity, Acoustic Waves and Device Applications (SPAWDA), Harbin, China, 2019.

[25] J. Yan, Z. Xu, Y. Wan, C. Chen, and X. Luo, "Consensus estimation-based target localization in underwater acoustic sensor networks," International Journal of Robust and Nonlinear Control, vol. 27, no. 9, pp. 1607-1627, 2017.

[26] H. Yang and B. Sikdar, "A mobility based architecture for underwater acoustic sensor networks," in IEEE GLOBECOM 2008 - 2008 IEEE Global Telecommunications Conference, New Orleans, LO, USA, 2008.

[27] A. Wang, J. Shen, C. Wang, H. Yang, and D. Liu, "Anonymous data collection scheme for cloud-aided mobile edge networks," Digital Communications and Networks, 2019.

[28] N. Cochard, J. L. Lacoume, P. Arzelies, and Y. Gabillet, "Underwater acoustic noise measurement in test tanks," IEEE Journal of Oceanic Engineering, vol. 25, no. 4, pp. 516-522, 2000.

[29] D. Pompili, T. Melodia, and I. F. Akyildiz, "Three-dimensional and two-dimensional deployment analysis for underwater acoustic sensor networks," Ad Hoc Networks, vol. 7, no. 4, pp. 778-790, 2009.

[30] W. Lin, D. Li, Y. Tan, J. Chen, and T. Sun, "Architecture of underwater acoustic sensor networks: a survey," in 2008 First International Conference on Intelligent Networks and Intelligent Systems, Wuhan, China, 2008.

[31] K. M. Awan, P. A. Shah, K. Iqbal, S. Gillani, W. Ahmad, and Y. Nam, "Underwater wireless sensor networks: a review of recent issues and challenges," Wireless Communications and Mobile Computing, vol. 2019, Article ID 6470359, 20 pages, 2019.

[32] N. Saeed, A. Celik, T. Y. Al-Naffouri, and M.-S. Alouini, "Underwater optical wireless communications, networking, and localization: a survey," Ad Hoc Networks, vol. 94, p. 101935, 2019.

[33] J. H. Cui, J. Kong, M. Gerla, and S. Zhou, "Challenges: building scalable and distributed underwater wireless sensor networks (UWSNs) for aquatic applications," Channels, vol. 45, no. 4, pp. 22-35, 2005.

[34] C. Lv, S. Wang, M. Tan, and L. Chen, "UA-MAC: an underwater acoustic channel access method for dense mobile 
underwater sensor networks," International Journal of Distributed Sensor Networks, vol. 10, no. 2, 2014.

[35] K. Chen, Y. Zhou, and J. He, "A localization scheme for underwater wireless sensor networks," International Journal of Advanced Science and Technology, vol. 4, 2009.

[36] Z. Zhou, Z. Peng, J.-H. Cui, Z. Shi, and A. Bagtzoglou, "Scalable localization with mobility prediction for underwater sensor networks," IEEE Transactions on Mobile Computing, vol. 10, no. 3, pp. 335-348, 2011.

[37] M. Erol-Kantarci, S. Oktug, L. Vieira, and M. Gerla, "Performance evaluation of distributed localization techniques for mobile underwater acoustic sensor networks," Ad Hoc Networks, vol. 9, no. 1, pp. 61-72, 2011.

[38] S. Capkun, M. Hamdi, and J.-P. Hubaux, "GPS-free positioning in " mobile ad hoc networks," Cluster Computing, vol. 5, no. 2, pp. 157-167, 2002.

[39] A. Caruso, S. Chessa, S. De, and A. Urpi, "GPS free coordinate assignment and routing in wireless sensor networks," in Proceedings IEEE 24th Annual Joint Conference of the IEEE Computer and Communications Societies, Miami, FL, USA, 2005.

[40] P. M. Ameer and L. Jacob, "Underwater localization using stochastic proximity embedding and multi-dimensional scaling," Wireless Networks, vol. 19, no. 7, pp. 1679-1690, 2013.

[41] Y. Zhou, K. Chen, J. He, J. Chen, and A. Liang, "A hierarchical localization scheme for large scale underwater wireless sensor networks," in 2009 11th IEEE International Conference on High Performance Computing and Communications, Seoul, South Korea, 2009.

[42] V. M. Janik, S. M. Parijs, and P. M. Thompson, "A twodimensional acoustic localization system for marine mammals," Marine Mammal Science, vol. 16, no. 2, pp. 437-447, 2000.

[43] T. Bian, R. Venkatesan, and C. Li, "Design and evaluation of a new localization scheme for underwater acoustic sensor networks," in GLOBECOM 2009 - 2009 IEEE Global Telecommunications Conference, Honolulu, HI, USA, 2009.

[44] T. Bian, R. Venkatesan, and C. Li, "An improved localization method using error probability distribution for underwater sensor networks," in 2010 IEEE International Conference on Communications, Cape Town, South Africa, 2010.

[45] D. Mirza and C. Schurgers, "Motion-aware self-localization for underwater networks," in Proceedings of the third ACM international workshop on Wireless network testbeds, experimental evaluation and characterization - WuWNeT '08, New York, NY, USA, 2008.

[46] V. Chandrasekhar and W. Seah, "An area localization scheme for underwater sensor networks," in OCEANS 2006 - Asia Pacific, Singapore, 2006.

[47] Y. Zhou, B. J. Gu, K. Chen, J. B. Chen, and H. B. Guan, “An range-free localization scheme for large scale underwater wireless sensor networks," Journal of Shanghai Jiaotong University, vol. 14, no. 5, pp. 562-568, 2009.

[48] W. Cheng, A. Y. Teymorian, L. Ma, X. Cheng, X. Lu, and $\mathrm{Z}$. Lu, "Underwater localization in sparse $3 \mathrm{D}$ acoustic sensor networks," in 2008 Proceedings IEEE INFOCOM - The 27th Conference on Computer Communications, Phoenix, AZ, USA, 2008.

[49] A. K. Othman, A. E. Adams, and C. C. Tsimenidis, "Node discovery protocol and localization for distributed underwater acoustic networks," in Advanced Int'l Conference on Telecommunications and Int'l Conference on Internet and Web Appli- cations and Services (AICT-ICIW'06), Guadelope, French Caribbean, French Caribbean, 2006.

[50] A.-K. Othman, "GPS-less localization protocol for underwater acoustic networks," in 2008 5th IFIP International Conference on Wireless and Optical Communications Networks (WOCN '08), Surabaya, Indonesia, 2008.

[51] D. Mirza and C. Schurgers, "Collaborative localization for fleets of underwater drifters," in OCEANS 2007, Vancouver, BC, Canada, 2007.

[52] V. Chandrasekhar, W. K. Seah, Y. S. Choo, and H. V. Ee, "Localization in underwater sensor networks: survey and challenges," in Proceedings of the 1st ACM international workshop on Underwater networks, pp. 33-40, Los Angeles, CA, USA, September 2006.

[53] M. Erol, L. F. M. Vieira, and M. Gerla, "AUV-aided localization for underwater sensor networks," in International Conference on Wireless Algorithms, Systems and Applications (WASA 2007), Chicago, IL, USA, 2007.

[54] D. D. Palma, F. Arrichiello, G. Parlangeli, and G. Indiveri, "Underwater localization using single beacon measurements: observability analysis for a double integrator system," Ocean Engineering, vol. 142, pp. 650-665, 2017.

[55] Z. Peng, J.-H. Cui, B. Wang, K. Ball, and L. Freitag, "An underwater network testbed: design, implementation and measurement," in Proceedings of the second workshop on Underwater networks - WuWNet '07, Montreal, Quebec, Canada, 2007.

[56] H. Luo, Y. Zhao, Z. Guo, S. Liu, P. Chen, and L. M. Ni, "UDB: using directional beacons for localization in underwater sensor networks," in 2008 14th IEEE International Conference on Parallel and Distributed Systems, Melbourne, VIC, Australia, 2008.

[57] X. Cheng, H. Shu, Q. Liang, and D. H.-C. Du, "Silent positioning in underwater acoustic sensor networks," IEEE Transactions on Vehicular Technology, vol. 57, no. 3, pp. 1756-1766, 2008.

[58] M. Erol, L. F. M. Vieira, and M. Gerla, "Localization with Dive'N'Rise (DNR) beacons for underwater acoustic sensor networks," in Proceedings of the second workshop on Underwater networks - WuWNet '07, New York, NY, USA, 2007.

[59] H. Luo, Z. Guo, W. Dong, F. Hong, and Y. Zhao, "LDB: localization with directional beacons for sparse 3D underwater acoustic sensor networks," Journal of Networks, vol. 5, no. 1, 2010.

[60] J. Luo, L. Fan, S. Wu, and X. Yan, "Research on localization algorithms based on acoustic communication for underwater sensor networks," Sensors, vol. 18, no. 2, p. 67, 2018.

[61] X. Cheng, A. Thaeler, G. Xue, and D. Chen, “TPS: a timebased positioning scheme for outdoor wireless sensor networks," in IEEE INFOCOM 2004, Hong Kong, China, 2004.

[62] H.-P. Tan, A. F. Gabor, Z. A. Eu, and W. K. G. Seah, “A wide coverage positioning system (WPS) for underwater localization," in 2010 IEEE International Conference on Communications, Cape Town, South Africa, 2010.

[63] X. Tan and J. Li, "Cooperative positioning in underwater sensor networks," IEEE Transactions on Signal Processing, vol. 58, no. 11, pp. 5860-5871, 2010.

[64] B. Fu, F. Zhang, M. Ito, Y. Watanabe, and T. Aoki, "Development of a new underwater positioning system based on sensor network," Artificial Life and Robotics, vol. 13, no. 1, pp. 45-49, 2008. 
[65] I. X. Gao, F. Zhang, and M. Ito, "Underwater acoustic positioning system based on propagation loss and sensor network," in 2012 Oceans - Yeosu, Yeosu, South Korea, 2012.

[66] D. Moreno, A. M. Pascoal, A. Alcocer, and J. Aranda, "Optimal sensor placement for underwater target positioning with noisy range measurements," IFAC Proceedings Volumes, vol. 43, no. 20, pp. 85-90, 2010.

[67] Y. Guo and Y. Liu, "Localization for anchor-free underwater sensor networks," Computers \& Electrical Engineering, vol. 39, no. 6, pp. 1812-1821, 2013.

[68] L. Emokpae and M. Younis, "Surface based anchor-free localization algorithm for underwater sensor networks," in 2011 IEEE International Conference on Communications (ICC), Kyoto, Japan, 2011.

[69] K. Ishii and N. Sato, "GPS-free host approaching in mobile ad-hoc networks," in 2013 Seventh International Conference on Innovative Mobile and Internet Services in Ubiquitous Computing, Taichung, Taiwan, 2013.

[70] Q. Fengzhong, W. Shiyuan, W. Zhihui, and L. Zubin, "A survey of ranging algorithms and localization schemes in underwater acoustic sensor network," China Communications, vol. 13, no. 3, pp. 66-81, 2016.

[71] Y. Guo, X. Kang, Q. Han, and J. Wang, "A localization algorithm for underwater wireless sensor networks based on ranging correction and inertial coordination," KSII Transactions on Internet and Information Systems, vol. 13, 2019.

[72] M. Zhou, Z. Zhong, and X. Fang, "Sensor-target geometry for hybrid bearing/range underwater localization," IFAC Proceedings Volumes, vol. 46, no. 20, pp. 724-729, 2013.

[73] J. Luo and L. Fan, "A two-phase time synchronization-free localization algorithm for underwater sensor networks," Sensors, vol. 17, no. 4, p. 726, 2017.

[74] S. Poursheikhali and H. Zamiri-Jafarian, "TDOA based target localization in inhomogenous underwater wireless sensor network," in 2015 5th International Conference on Computer and Knowledge Engineering (ICCKE), Mashhad, Iran, 2015.

[75] R. M. Vaghefi and R. M. Buehrer, "Asynchronous time-ofarrival-based source localization," in 2013 IEEE International Conference on Acoustics, Speech and Signal Processing, Vancouver, BC, Canada, 2013.

[76] Y. Zou and Q. Wan, "Asynchronous time-of-arrival-based source localization with sensor position uncertainties," IEEE Communications Letters, vol. 20, no. 9, pp. 1860-1863, 2016.

[77] I. Ullah, J. Chen, X. Su, C. Esposito, and C. Choi, "Localization and detection of targets in underwater wireless sensor using distance and angle based algorithms," IEEE Access, vol. 7, pp. 45693-45704, 2019.

[78] R. Diamant and L. Lampe, "Underwater localization with time synchronization and propagation speed uncertainties," IEEE Transactions on Mobile Computing, vol. 12, no. 7, pp. 1257-1269, 2013.

[79] J. Yi, D. Mirza, R. Kastner, C. Schurgers, P. Roberts, and J. Jaffe, "ToA-TS: time of arrival based joint time synchronization and tracking for mobile underwater systems," Ad Hoc Networks, vol. 34, pp. 211-223, 2015.

[80] W. A. P. van Kleunen, K. C. H. Blom, N. Meratnia, A. B. J. Kokkeler, P. J. M. Havinga, and G. J. M. Smit, "Underwater localization by combining time-of-flight and direction-ofarrival," in OCEANS 2014 - TAIPEI, Taipei, Taiwan, 2014.
[81] D. Mirza, P. Naughton, C. Schurgers, and R. Kastner, "Realtime collaborative tracking for underwater networked systems," Ad Hoc Networks, vol. 34, pp. 196-210, 2015.

[82] J. Liu, Z. Wang, J. H. Cui, S. Zhou, and B. Yang, "A joint time synchronization and localization design for mobile underwater sensor networks," IEEE Transactions on Mobile Computing, vol. 15, no. 3, pp. 530-543, 2016.

[83] P. Carroll, S. Zhou, H. Zhou, J.-H. Cui, and P. Willett, "Underwater localization based on multicarrier waveforms," in OCEANS 2010 MTS/IEEE SEATTLE, Seattle, WA, USA, 2010.

[84] P. Carroll, S. Zhou, H. Zhou, X. Xu, J. H. Cui, and P. Willett, "Underwater localization and tracking of physical systems," Journal of Electrical and Computer Engineering, vol. 2012, Article ID 683919, 11 pages, 2012.

[85] P. Rong and M. L. Sichitiu, "Angle of arrival localization for wireless sensor networks," in 2006 3rd Annual IEEE Communications Society on Sensor and Ad Hoc Communications and Networks, Reston, VA, USA, 2006.

[86] J. B. Andersen and K. I. Pedersen, "Angle-of-arrival statistics for low resolution antennas," IEEE Transactions on Antennas and Propagation, vol. 50, no. 3, pp. 391-395, 2002.

[87] A. Mesmoudi, M. Feham, and N. Labraoui, "Wireless sensor networks localization algorithms: a comprehensive survey," International journal of Computer Networks \& Communications, vol. 5, no. 6, pp. 45-64, 2013.

[88] Q. H. Spencer, B. D. Jeffs, M. A. Jensen, and A. L. Swindlehurst, "Modeling the statistical time and angle of arrival characteristics of an indoor multipath channel," IEEE Journal on Selected Areas in Communications, vol. 18, no. 3, pp. 347360, 2000.

[89] K. Tong, X. Wang, A. Khabbazibasmenj, and A. Dounavis, "RSS-based localization in obstructed environment with unknown path loss exponent," in 2014 IEEE 80th Vehicular Technology Conference (VTC2014-Fall), Vancouver, BC, Canada, 2014

[90] T. L. N. Nguyen and Y. Shin, "An efficient RSS localization for underwater wireless sensor networks," Sensors, vol. 19, no. 14, p. 3105, 2019.

[91] T. Xu, Y. Hu, B. Zhang, and G. Leus, "RSS-based sensor localization in underwater acoustic sensor networks," in 2016 IEEE International Conference on Acoustics, Speech and Signal Processing (ICASSP), Shanghai, China, 2016.

[92] M. Hosseini, H. Chizari, C. K. Soon, and R. Budiarto, "RSSbased distance measurement in underwater acoustic sensor networks: an application of the Lambert W function," in 2010 4th International Conference on Signal Processing and Communication Systems, Gold Coast, QLD, Australia, 2010.

[93] B. Zhang, H. Wang, T. Xu, L. Zheng, and Q. Yang, "Received signal strength-based underwater acoustic localization considering stratification effect," in OCEANS 2016 - Shanghai, Shanghai, China, 2016.

[94] S. Poursheikhali and H. Zamiri-Jafarian, "Ranging in underwater wireless sensor network: received signal strength approach," in 2016 IEEE Wireless Communications and Networking Conference, Doha, Qatar, 2016.

[95] Y.-s. Yan, H.-y. Wang, X.-h. Shen, F.-z. Yang, and Z. Chen, "Efficient convex optimization method for underwater passive source localization based on RSS with WSN," in 2012 IEEE International Conference on Signal Processing, Communication and Computing (ICSPCC 2012), Hong Kong, China, 2012. 
[96] D. Park, J. Kim, and W. K. Chung, "Simulated 3D underwater localization based on RF sensor model using EKF," in 2011 8th International Conference on Ubiquitous Robots and Ambient Intelligence (URAI), Incheon, South Korea, 2011.

[97] J. Gao, X. Shen, and H. Wang, "A hybrid localization algorithm for multi-hop mobile underwater acoustic networks," Journal of Marine Science and Technology, vol. 22, no. 3, pp. 494-500, 2017.

[98] W. Wang, S. Li, J. Yang, Z. Liu, and W. Zhou, "Feature extraction of underwater target in auditory sensation area based on MFCC," in 2016 IEEE/OES China Ocean Acoustics (COA), Harbin, China, 2016.

[99] T. S. Tiang and M. N. Mahyuddin, "Cooperative formation control algorithm of a generic multi-agent system applicable for multiautonomous surface vehicles," in 2016 IEEE International Conference on Underwater System Technology: Theory and Applications (USYS), Penang, Malaysia, 2016.

[100] D. Muhammed, M. Anisi, M. Zareei, C. Vargas-Rosales, and A. Khan, "Game theory-based cooperation for underwater acoustic sensor networks: taxonomy, review, research challenges and directions," Sensors, vol. 18, no. 2, p. 425, 2018.

[101] S. Ashraf, M. Gao, Z. Chen, S. K. Haider, and Z. Raza, "Efficient node monitoring mechanism in WSN using contikimac protocol," International Journal of Advanced Computer Science and Applications, vol. 8, no. 11, pp. 429-437, 2017.

[102] H. Li, Y. He, X. Cheng, H. Zhu, and L. Sun, "Security and privacy in localization for underwater sensor networks," IEEE Communications Magazine, vol. 53, no. 11, pp. 56-62, 2015. 


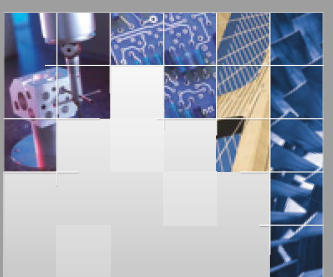

\section{Enfincering}
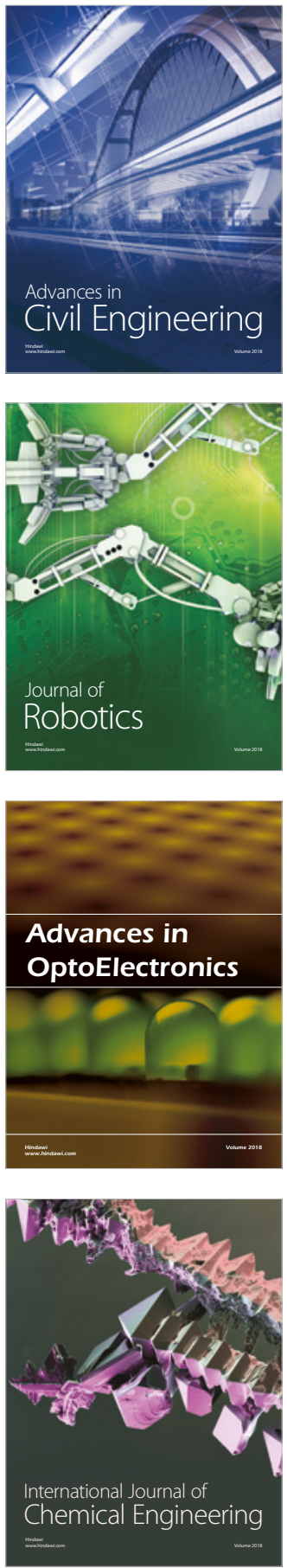

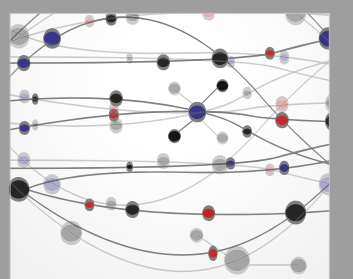

\section{Rotating \\ Machinery}

The Scientific World Journal

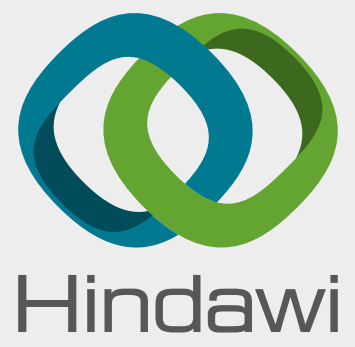

Submit your manuscripts at

www.hindawi.com
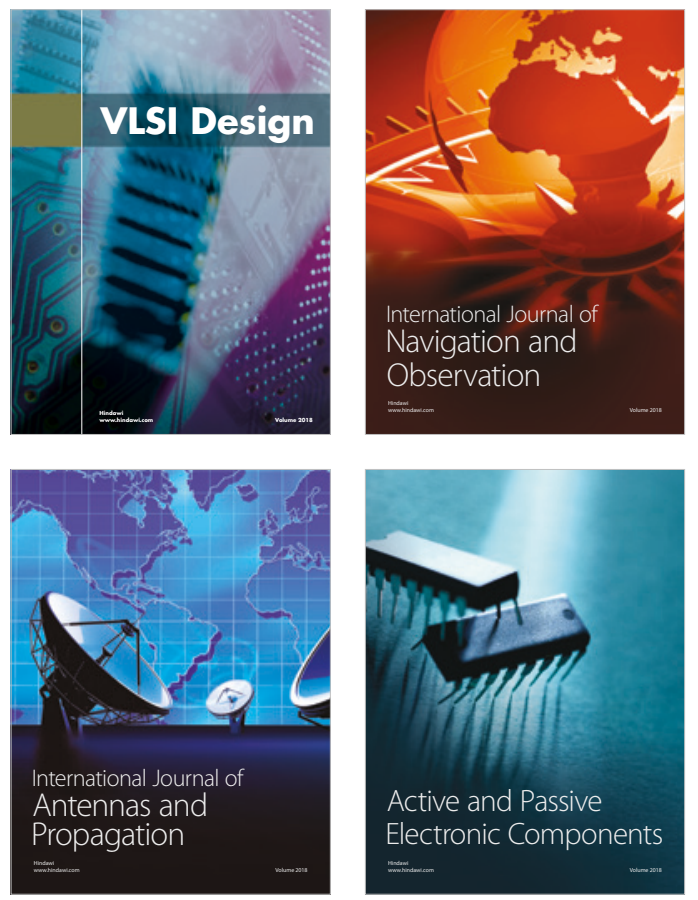
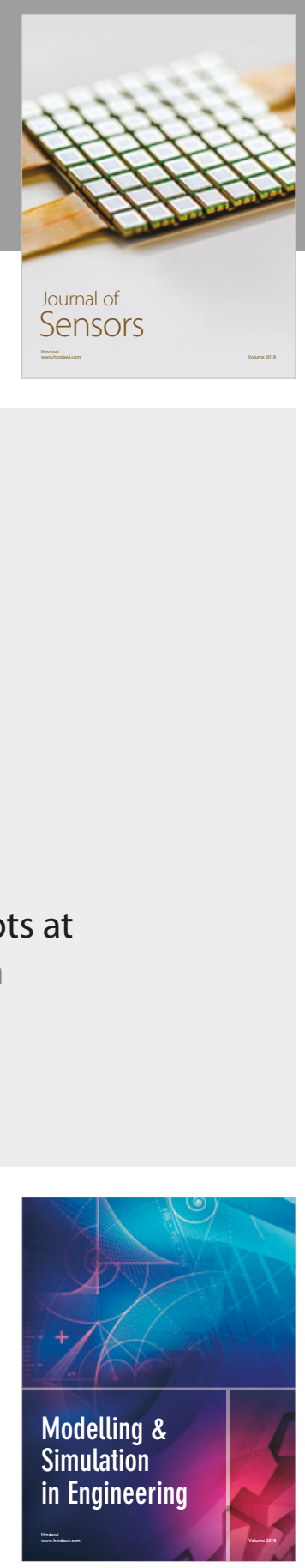

\section{Advances \\ Multimedia}
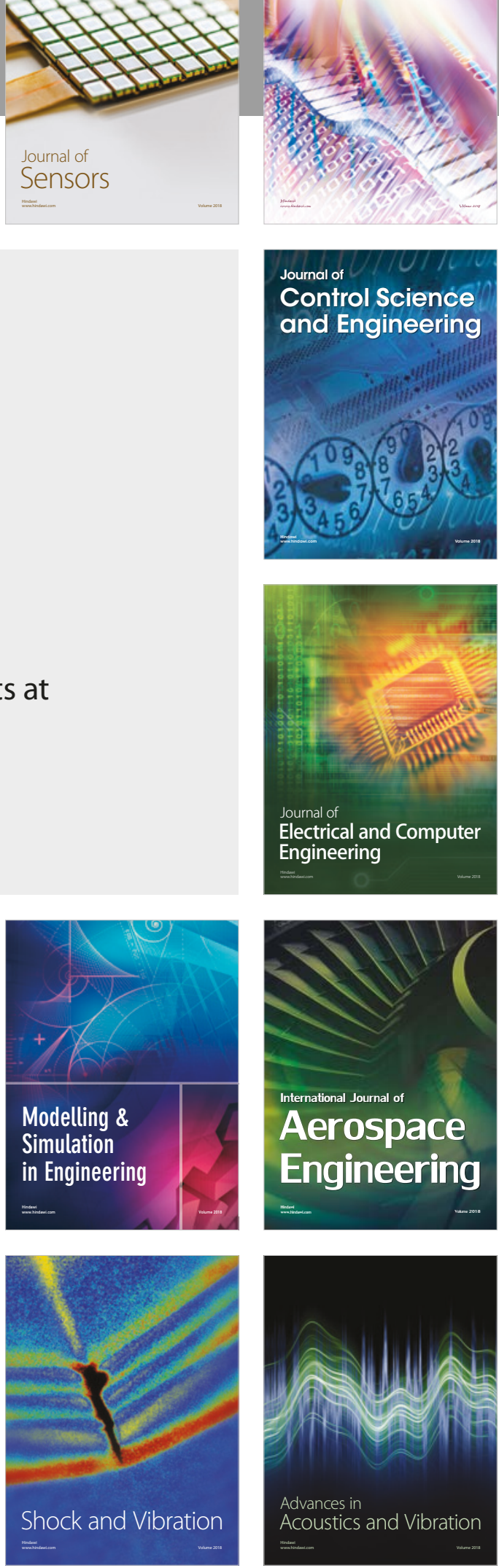